\title{
Radiative transfer modeling through terrestrial atmosphere and ocean accounting for inelastic processes: Software package SCIATRAN
}

\author{
V.V. Rozanov ${ }^{\mathrm{a}}$ T. Dinter ${ }^{\mathrm{a}, \mathrm{b}, *}$, A.V. Rozanov ${ }^{\mathrm{a}}$ A. Wolanin ${ }^{\mathrm{a}, \mathrm{b}}$, A. Bracher ${ }^{\mathrm{a}, \mathrm{b}}$, J.P. Burrows ${ }^{\mathrm{a}}$ \\ a Institute of Remote Sensing, University of Bremen, Otto Hahn Allee 1, D-28334 Bremen, Germany \\ ${ }^{\mathrm{b}}$ Alfred-Wegener-Institute Helmholtz Centre for Polar and Marine Research, Bussestraße 24, D-27570 Bremerhaven, Germany
}

\section{A R T I C L E I N F O}

\section{Article history:}

Received 18 November 2016

Received in revised form

3 March 2017

Accepted 3 March 2017

Available online 6 March 2017

Keywords:

Radiative transfer

Coupled ocean-atmosphere

Inelastic scattering

Vibrational Raman scattering

Fluorescence

SCIATRAN

\begin{abstract}
A B S T R A C T
SCIATRAN is a comprehensive software package which is designed to model radiative transfer processes in the terrestrial atmosphere and ocean in the spectral range from the ultraviolet to the thermal infrared $(0.18-40 \mu \mathrm{m})$. It accounts for multiple scattering processes, polarization, thermal emission and oceanatmosphere coupling. The main goal of this paper is to present a recently developed version of SCIATRAN which takes into account accurately inelastic radiative processes in both the atmosphere and the ocean. In the scalar version of the coupled ocean-atmosphere radiative transfer solver presented by Rozanov et al. [61] we have implemented the simulation of the rotational Raman scattering, vibrational Raman scattering, chlorophyll and colored dissolved organic matter fluorescence.

In this paper we discuss and explain the numerical methods used in SCIATRAN to solve the scalar radiative transfer equation including trans-spectral processes, and demonstrate how some selected radiative transfer problems are solved using the SCIATRAN package. In addition we present selected comparisons of SCIATRAN simulations with those published benchmark results, independent radiative transfer models, and various measurements from satellite, ground-based, and ship-borne instruments.

The extended SCIATRAN software package along with a detailed User's Guide is made available for scientists and students, who are undertaking their own research typically at universities, via the web page of the Institute of Environmental Physics (IUP), University of Bremen: http://www.iup.physik.unibremen.de.
\end{abstract}

(c) 2017 Elsevier Ltd. All rights reserved.

\section{Contents}

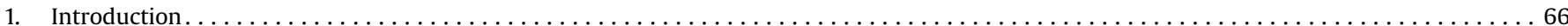

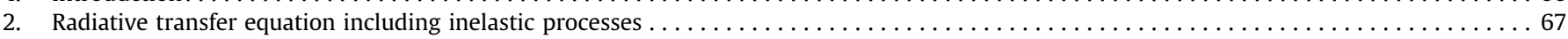

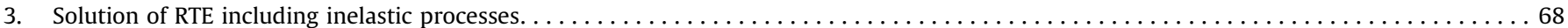

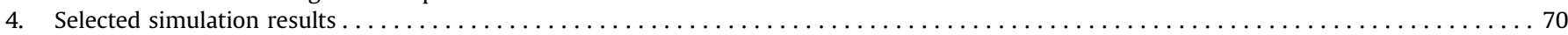

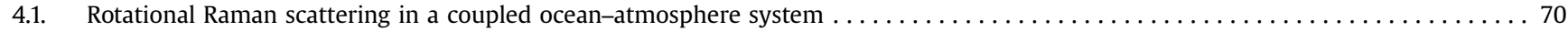

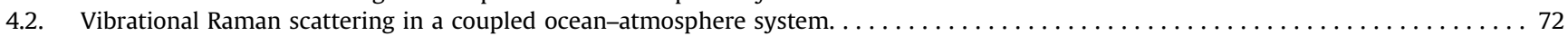

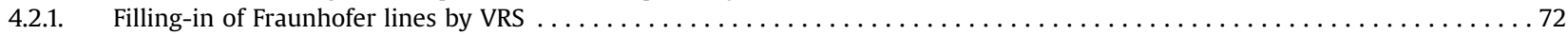

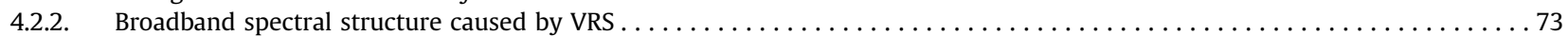

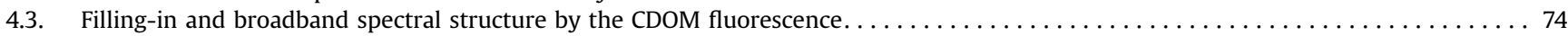

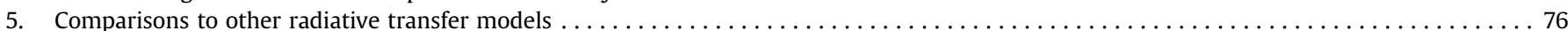

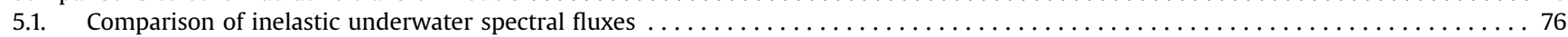

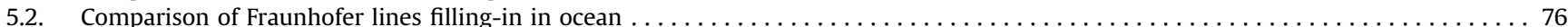

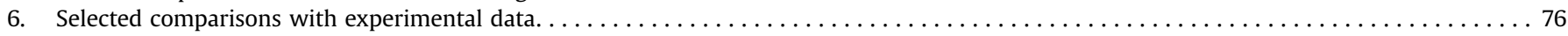

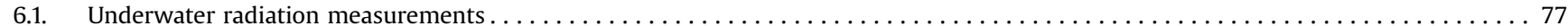

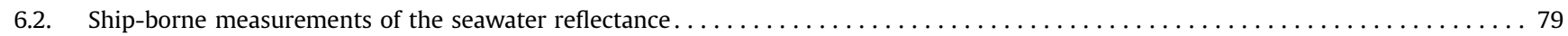

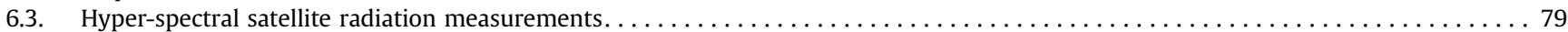

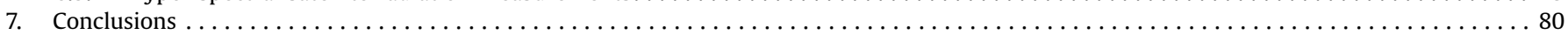

\footnotetext{
* Corresponding author at: Alfred-Wegener-Institute Helmholtz Centre for Polar and Marine Research, Bussestraße 24, D-27570 Bremerhaven, Germany.

E-mail addresses: rozanov@iup.physik.uni-bremen.de (V.V. Rozanov), tdinter@awi.de (T. Dinter).
} 


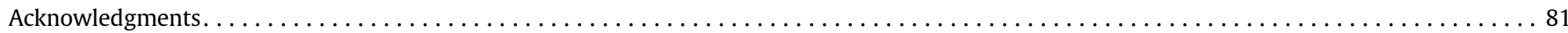

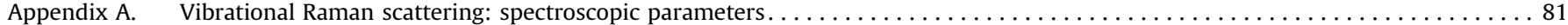

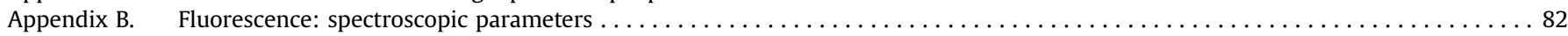

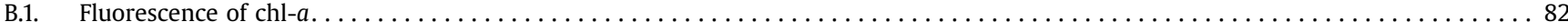

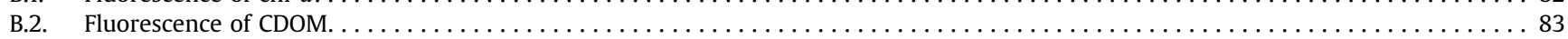

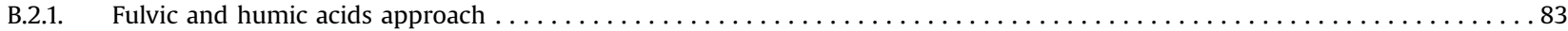

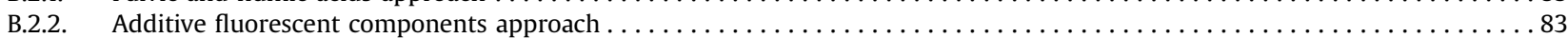

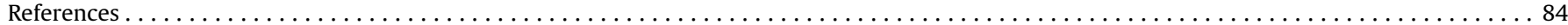

\section{Introduction}

The importance of inelastic radiative processes in the atmosphere and ocean has been addressed by many authors $[22,38,45,53,73,75,77]$. The most important of these processes are the rotational Raman scattering (RRS), vibrational Raman scattering (VRS) and fluorescence by chlorophyll $a$ (chl- $a$ ) and colored dissolved organic matter (CDOM).

In contrast to elastic scattering processes where the incident photons, after interaction with matter (gas, liquid, and solid), preserve the energy, in inelastic processes the frequency of a photon after interaction is shifted to red or blue. A red shift can be observed when part of the energy of the photon is transferred to the interacting matter. This process is referred to as the Stokes Raman scattering. The blue shift occurs when internal energy of the matter is transferred to the photon. This process is known as anti-Stokes Raman scattering [44]. If the energy change of the scattered photon is caused by a change of rotational or vibrational states of molecules, the Raman scattering process is referred to as the rotational (RRS) or vibrational Raman scattering (VRS), respectively. The rotational Raman scattering is important in air but not present in the water, whereas the vibrational Raman scattering is significant in water but of less importance in the air.

The rotational Raman scattering in the atmosphere results predominantly from photons being inelastically scattered by nitrogen and oxygen molecules. The contribution from water vapor and the atmospheric trace gases is in comparison to that of molecular oxygen and nitrogen negligibly small. The width of Stokes and anti-Stokes bands, which comprise numerous narrow rotational lines, is $\sim 1.2 \mathrm{~nm}$ at the wavelength of $400 \mathrm{~nm}$. Owing to the red and blue shift, RRS distributes the intensity of the scattered photons over several nanometer. The loss of intensity is proportional to the local intensity of the radiation field whereas the gain is proportional to the intensity at neighboring wavelengths. This leads for example to a filling-in of Fraunhofer lines which was independently discovered by Shefov [66] and Grainger and Ring [26] and is known as the Ring effect.

The VRS phenomenon results from the thermal vibrational properties of the water allowing the energy to be transferred to a specific vibrational mode of the $\mathrm{OH}$ bond. For a given pair of energy levels, the energy difference is expressed in terms of a constant frequency shift, e.g., $\sim 3400 \mathrm{~cm}^{-1}$ for the $\mathrm{OH}$ stretch vibrational mode in water [2]. For example, an incident wavelength of $\sim 400 \mathrm{~nm}$ results in the inelastically scattered light in the Stokes band having a wavelength of about $460 \mathrm{~nm}$, i.e., the VRS produces a wavelength shift of about $60 \mathrm{~nm}$ in the visible spectral range. The width of the Stokes band in this case is $\sim 8 \mathrm{~nm}[89,37]$. It is much larger than the wavelength shift due to RRS on $\mathrm{N}_{2}$ and $\mathrm{O}_{2}$ molecules in the air and also much larger than the width of any Fraunhofer line. In comparison to the Stokes VRS band, the antiStokes band is usually much less intense at the ambient temperatures of the world's ocean because it is associated with the relaxation of exited vibrational states, which are not significantly populated at atmospheric and oceanic temperatures [73].
Another well known inelastic process in the ocean is the fluorescence of chl- $a$ and CDOM. Fluorescence arises, when an absorbed photon excites an electron of a molecule which then can be transferred to a lower quantum state by undergoing interaction within the molecule or collision processes with other molecules. Thereafter the electron relaxes back to its original state and the emitted photon has lower energy than the original one. In contrast to the RRS and VRS effects, fluorescence occurs only at a lower quantum energy and longer wavelength than the originally absorbed photon. The spectral width of the fluorescence emission bands is in the range $25-100 \mathrm{~nm}$ depending on the fluorescence molecule $[28,30,71,79]$.

Inelastic radiative processes such as RRS, VRS, or fluorescence redistribute photons over wavelengths. This results in a filling in of solar Fraunhofer lines, as well as of trace gas absorption structures. This changes the spectral distribution of scattered light in the atmosphere and ocean. Photon redistribution caused by RRS affects atmospheric trace gas retrievals from measurements of scattered light with space-borne instrumentation significantly [80,85,58]. Furthermore, measurements of the filling-in of Fraunhofer lines may be used to estimate cloud top pressure from satellite measurements $[33,16]$ and aerosol properties from both surface and satellite observations $[87,88]$.

The impact of VRS on back-scattered light is similar to that of RRS. Vountas et al. [84] studied the impact of VRS on trace gas retrievals from the GOME satellite instrument and found that neglecting VRS results in significant errors in the DOAS (Differential Optical Absorption Spectroscopy) analysis, e.g., more than $30 \%$ for BrO slant columns over clear ocean scenarios. Grossmann et al. [27] included a VRS spectrum to improve their MAX-DOAS (Multi-AXis Differential Optical Absorption Spectroscopy) fit of IO in the marine boundary layer over the ocean especially close to the horizon. A comprehensive study of VRS impacting the $\mathrm{NO}_{2}$ retrieval was performed by Peters et al. [54].

The VRS spectral features provide useful information about energy transport in the atmosphere and ocean. As recently demonstrated by Dinter et al. [17] the VRS signal retrieved from hyperspectral satellite data can be used as a proxy for the light availability (scalar irradiance) in the global ocean, which is an important parameter for both estimating phytoplankton primary production from satellite data and assessing the heat content in the surface ocean.

In the UV spectral range, the filling-in of Fraunhofer lines by VRS in the ocean is decreased with increasing phytoplankton and CDOM concentration as they both absorb UV radiation. This characteristic was used to retrieve the oceanic chlorophyll content by Joiner et al. [35].

The information content of the filling-in signal originating from CDOM and chl- $a$ fluorescence was studied among others by Wolanin et al. $[91,90]$. In particular, it was demonstrated that the information from chlorophyll fluorescence measurements can be used to assess the physiological state of phytoplankton to further improve the knowledge of biogeochemical cycles, productivity and physiology of marine environments $[4,18]$, whereas the CDOM-fluorescence signal 
is useful to characterize CDOM composition and concentration independent from amount of chlorophyll [14]. To identify the filling-in of Fraunhofer lines by RRS, VRS, fluorescence of CDOM and chl- $a$ in hyperspectral satellite data, radiative transfer models able to simulate these processes in coupled oceanic-atmospheric system are necessary. At present, there are several radiative transfer codes able to simulate the rotational Raman scattering in the Earth's atmosphere $[34,43,46,61,81,85,86]$.

Sathyendranath and Platt [63] have implemented VRS in the quasi-single-scattering approximation reported in [23]. This two stream irradiance model was extended by Marshall and Smith [45] to include Raman scattering. Vountas et al. [84] incorporated the ocean reflectance model of Sathyendranath and Platt [63] into the radiative transfer code GOMETRAN [60]. Schröder et al. [64] incorporated VRS and fluorescence emission into a coupled oceanatmosphere radiative transfer model [20] based onto the matrix operator method. Monte Carlo simulations were performed by many authors $[37,73,74]$. The widely used Hydrolight software (www.hydrolight.info) also incorporates VRS and chlorophyll and CDOM fluorescence in its underwater radiative transfer calculations [49].

To provide the basis for the development and improvement of algorithms to retrieve atmospheric and oceanic constituents, whose retrieval is impacted by the inelastic processes, we have implemented RRS, VRS, fluorescence of chl- $a$ and CDOM into the coupled ocean-atmosphere radiative transfer model SCIATRAN [61]. To our knowledge, the new version of SCIATRAN model is the only freely available software code capable of modeling the radiative transfer through a coupled ocean-atmosphere system accounting for rotational Raman scattering, vibrational Raman scattering, as well as fluorescence of chl- $a$ and CDOM.

The paper is structured as follows. The formulation of the radiative transfer equation in the coupled ocean-atmosphere system including inelastic sources is given in Section 2. Subsequently, in Section 3, we briefly consider the numerical techniques used to solve formulated equations. Section 4 describes selected results obtained with the SCIATRAN model. Comparisons of SCIATRAN results with simulations by other radiative transfer models and measurement data are given in Sections 5 and 6, respectively. Appendix summarizes all implementation details.

\section{Radiative transfer equation including inelastic processes}

Radiative transfer processes in the terrestrial atmosphere and ocean can be considered as a standard boundary value problem (BVP) [12]. Restricting the consideration to the scalar case, the radiative transfer equation (RTE) is written as follows:

$\mu \frac{\partial I_{\mathrm{s}}(\tau, \Omega, \lambda)}{\partial \tau}=-I_{\mathrm{s}}(\tau, \Omega, \lambda)+J_{\mathrm{s}}(\tau, \Omega, \lambda), \quad s=1,2$.

Here, $\tau \in\left[0, \tau_{0}\right]$ is the optical depth changing from 0 at the top of the plane-parallel medium to $\tau_{0}$ at the bottom, the angular variable $\Omega:=\{\mu, \varphi\}$ describes the set of variables $\mu \in[-1,1]$ and $\varphi \in[0,2 \pi], \mu$ is the cosine of the polar angle $\vartheta$ as measured from the positive $\tau$-axis (negative $z$-axis) and $\varphi$ is the azimuthal angle, measured from the positive $x$-axis of the basic Cartesian coordinate system in the clockwise direction when looking upward, $I_{\mathrm{S}}(\tau, \Omega, \lambda)$ is the total intensity (or radiance) at the optical depth $\tau$ in the direction $\Omega$ and the wavelength $\lambda, J_{\mathrm{s}}(\tau, \Omega, \lambda)$ is the source function, the subscript $s$ will be set to 1 or 2 to denote optical parameters and radiation fields in the atmosphere and the ocean, respectively.

We assume that the atmosphere is illuminated by unidirectional $\left(\mu_{0}, \varphi_{0}\right)$ solar light beam, $\mu_{0}$ and $\varphi_{0}$ are the cosine of the solar zenith angle and azimuthal angle, respectively. The solar zenith angle is defined as an angle between the positive direction of $z$ axis and the direction to the sun. The $x$-axis of basic Cartesian coordinate system is chosen so that it points opposite to the sun. Therefore, the azimuthal angle of the solar beam equals to zero $\left(\varphi_{0}=0\right)$. Thus, the upper boundary condition for Eq. (1) is given by $I_{1}(0, \Omega, \lambda)=F_{0}(\lambda) \delta\left(\Omega-\Omega_{0}\right), \quad \mu>0$,

where $F_{0}(\lambda)$ is the extraterrestrial solar flux through unit area normal to the solar beam at the top of atmosphere and $\delta\left(\Omega-\Omega_{0}\right)=\delta\left(\mu-\mu_{0}\right) \delta\left(\varphi-\varphi_{0}\right)$ is the Dirac delta function (see, e.g., $[41])$.

The lower boundary condition describes the reflection of radiation at the ocean bottom and is given by

$I_{2}\left(\tau_{0}, \Omega, \lambda\right)=\frac{1}{\pi} \int_{\Omega^{+}} R_{2}\left(\Omega, \Omega^{\prime}\right) I_{2}\left(\tau_{0}, \Omega^{\prime}, \lambda\right) \mu^{\prime} d \Omega^{\prime}, \quad \mu<0$,

where $\Omega^{+}$denotes that the angular integration is performed over positive $\mu^{\prime}$ and $\varphi^{\prime} \in[0,2 \pi] . R_{2}\left(\Omega, \Omega^{\prime}\right)$ determines angular reflection properties of the underlying ocean bottom.

In the case of a coupled ocean-atmosphere system there is also an interface between ocean and atmosphere where specific conditions need to be formulated. In particular, it is assumed that at the air-water interface the reflection and transmission of the radiation occur according to Fresnel's law [7]. Under this assumption the intensity of the upwelling radiation at the bottom of the atmosphere is given by

$I_{1}\left(\tau_{a}, \Omega, \lambda\right)=\mathcal{R}_{\mathrm{a}} I_{1}\left(\tau_{a}, \Omega^{\prime}, \lambda\right)+\mathcal{T}_{\text {wa }} I_{2}\left(\tau_{a}, \Omega^{\prime}, \lambda\right), \quad \mu<0$.

where $\tau_{a}$ is the optical thickness of the atmosphere, and linear integral operators $\mathcal{R}_{\mathrm{a}}$ and $\mathcal{T}_{\text {wa }}$ are

$\mathcal{R}_{\mathrm{a}}=\int_{\Omega^{+}} d \Omega^{\prime} R_{\mathrm{a}}\left(\Omega, \Omega^{\prime}\right) \otimes, \quad \mathcal{T}_{\mathrm{wa}}=\int_{\Omega^{-}} d \Omega^{\prime} T_{\mathrm{wa}}\left(\Omega, \Omega^{\prime}\right) \otimes$,

$\Omega^{-}$denotes that the angular integration is performed over negative $\mu^{\prime}$ and $\varphi^{\prime} \in[0,2 \pi]$. The first term in the right-hand side of Eq. (4) describes the radiation reflected from the ocean-atmosphere interface back to the atmosphere and second term represents the radiation transmitted from the ocean to the atmosphere (the socalled water-leaving radiation), $R_{\mathrm{a}}$ and $T_{\mathrm{wa}}$ are known as reflection and transmission functions.

The intensity of the downwelling radiation at the top of the ocean is written as follows:

$I_{2}\left(\tau_{a}, \Omega, \lambda\right)=\mathcal{R}_{\mathrm{w}} I_{2}\left(\tau_{a}, \Omega^{\prime}, \lambda\right)+\mathcal{T}_{\mathrm{aw}} I_{1}\left(\tau_{a}, \Omega^{\prime}, \lambda\right), \quad \mu>0$

Here, the first term in the right-hand side describes the radiation reflected from the ocean-atmosphere interface back to the ocean, and the second term represents the radiation transmitted from the atmosphere to the ocean, the linear integral operators $\mathcal{R}_{\mathrm{w}}$ and $\mathcal{T}_{\text {aw }}$ are defined similarly to Eq. (5) where $R_{\mathrm{a}}$ and $T_{\mathrm{wa}}$ should be replaced by known reflection and transmission functions $R_{\mathrm{w}}$ and $T_{\mathrm{aw}}$, respectively, also the integral limits $\Omega^{+}$and $\Omega^{-}$should be exchanged. Reflection and transmission functions involved in the interface conditions given by Eqs. (4) and (6) are implemented for absolutely flat and wind-roughened air-water interface including shadowing effects and Gaussian distribution of wave slopes [52]. Sea surface reflection and transmission, including polarization, also can be computed using ray tracing and randomly generated sea surfaces [50]. Such calculations automatically incorporate also wave shadowing effects.

Source functions $J_{1}(\tau, \Omega, \lambda)$ and $J_{2}(\tau, \Omega, \lambda)$ in Eq. (1) describe radiation sources within the atmosphere and ocean, respectively. The source function in the atmosphere is used in the following form:

$J_{1}(\tau, \Omega, \lambda)=\frac{\omega_{1}(\lambda)}{4 \pi} \int_{\Omega} \mathcal{P}_{1}\left(\Omega, \Omega^{\prime}\right) I_{1}\left(\tau, \Omega^{\prime}, \lambda\right) d \Omega^{\prime}+\mathcal{J}_{1}(\tau, \Omega, \lambda)$, 
where the first and second terms describe the multiple scattering source function of elastic and inelastic processes in the atmosphere, respectively $[62,80,85]$. In particular, the elastic scattering processes include the Rayleigh scattering as well as scattering by aerosol and cloud particles. The optical parameters of the atmosphere, i.e., the total scattering coefficient, $\beta_{1}(\lambda)$, weighted phase function, $\mathcal{P}_{1}\left(\Omega, \Omega^{\prime}\right)$, single scattering albedo, $\omega_{1}(\lambda)$, and extinction coefficient, $e_{1}(\lambda)$, are given by

$\beta_{1}(\lambda)=\beta_{\mathrm{cab}}(\lambda)+\beta_{\text {aer }}(\lambda)+\beta_{\text {cld }}(\lambda)$,

$\mathcal{P}_{1}=\frac{1}{\beta_{1}(\lambda)}\left[\beta_{\mathrm{cab}}(\lambda) \mathcal{P}_{\mathrm{cab}}+\beta_{\mathrm{aer}}(\lambda) \mathcal{P}_{\mathrm{aer}}+\beta_{\mathrm{cld}}(\lambda) \mathcal{P}_{\mathrm{cld}}\right]$

$\omega_{1}(\lambda)=\beta_{1}(\lambda) / e_{1}(\lambda)$

$e_{1}(\lambda)=a_{1}(\lambda)+\beta_{\text {ray }_{1}}(\lambda)+\beta_{\text {aer }}(\lambda)+\beta_{\text {cld }}(\lambda)$,

where $a_{1}(\lambda)$ is an absorption coefficient comprising absorption by gaseous components as well as by aerosol and cloud particles. All optical parameters of the atmosphere might vary with the vertical coordinate (optical depth). However, for the sake of brevity we do not indicate this dependence explicitly. We note that here the Cabannes scattering coefficient $\beta_{\mathrm{cab}}(\lambda)$ and Cabannes phase function $\mathcal{P}_{\text {cab }}$ are used instead of the classical Rayleigh to describe the scattering of photons without frequency shift whereas the extinction coefficient $e_{1}(\lambda)$ includes the classical Rayleigh scattering coefficient $\beta_{\text {ray }_{1}}(\lambda)$ which accounts for both attenuation processes caused by the scattering in the spatial and spectral domains (see [62] for details).

The inelastic scattering source function $\mathcal{J}_{1}(\tau, \Omega, \lambda)$ (second term in the right-hand side of Eq. (7)) describes the contribution of the rotational Raman scattering which is given as

$\mathcal{J}_{1}(\tau, \Omega, \lambda)=\frac{\omega_{1}(\lambda)}{4 \pi} \sum_{j=1}^{L} \int_{\Omega} \mathcal{P}_{1}^{\text {in }}\left(\Omega, \Omega^{\prime} ; \lambda_{j}, \lambda\right) I_{1}\left(\tau, \Omega^{\prime}, \lambda_{j}\right) d \Omega^{\prime}$,

where $L$ is the number of Stokes and anti-Stokes spectral lines taken into account, $\mathcal{P}_{1}^{\text {in }}\left(\Omega, \Omega^{\prime} ; \lambda_{j}, \lambda\right)$ is spatial-frequency phase function given by

$\mathcal{P}_{1}^{\mathrm{in}}\left(\Omega, \Omega^{\prime} ; \lambda_{j}, \lambda\right)=\frac{r_{\mathrm{r}}\left(\lambda, \lambda_{\mathrm{j}}\right) \mathcal{P}_{\mathrm{rrs}}\left(\Omega, \Omega^{\prime}\right)}{\beta_{1}(\lambda)}$,

$r_{\mathrm{r}}\left(\lambda, \lambda_{j}\right)$ and $\mathcal{P}_{\mathrm{rrs}}\left(\Omega, \Omega^{\prime}\right)$ are the rotational Raman scattering coefficient and the phase function, respectively. Details of the implementation are given in $[62,85]$.

The source function in the ocean is used in the following form:

$J_{2}(\tau, \Omega, \lambda)=\frac{\omega_{2}(\lambda)}{4 \pi} \int_{\Omega} \mathcal{P}_{2}\left(\Omega, \Omega^{\prime}\right) I_{2}\left(\tau, \Omega^{\prime}, \lambda\right) d \Omega^{\prime}+\mathcal{J}_{2}(\tau, \Omega, \lambda)$.

The first term in this equation describes the elastic scattering processes in the ocean which include pure water scattering and scattering by particular matter. The total scattering coefficient, $\beta_{2}(\lambda)$, weighted phase function, $\mathcal{P}_{2}\left(\Omega, \Omega^{\prime}\right)$, single scattering albedo, $\omega_{2}(\lambda)$, and total extinction coefficient, $e_{2}(\lambda)$ are given as

$\beta_{2}(\lambda)=\beta_{\text {ray }_{2}}(\lambda)+\sum_{k=1}^{N_{p}} \beta_{k}(\lambda)$
$\mathcal{P}_{2}=\frac{1}{\beta_{2}(\lambda)}\left[\beta_{\text {ray }_{2}}(\lambda) \mathcal{P}_{\text {ray }}+\sum_{k=1}^{N_{p}} \beta_{\mathrm{k}}(\lambda) \mathcal{P}_{\mathrm{k}}\right]$

$\omega_{2}(\lambda)=\frac{\beta_{2}(\lambda)}{e_{2}(\lambda)}, \quad e_{2}(\lambda)=a_{2}(\lambda)+\beta_{2}(\lambda)+\beta_{\mathrm{vrs}}(\lambda)+\sum_{\mathrm{f}=1}^{N_{\mathrm{f}}} a_{\mathrm{f}}(\lambda)$

where $\beta_{\text {ray }_{2}}(\lambda)$ and $\mathcal{P}_{\text {ray }}\left(\Omega, \Omega^{\prime}\right)$ are the scattering coefficient and phase function of pure sea water caused by the density fluctuations, $N_{p}$ is the number of particular matter components having scattering coefficient $\beta_{\mathrm{k}}(\lambda)$ and phase function $\mathcal{P}_{\mathrm{k}}\left(\Omega, \Omega^{\prime}\right), a_{2}(\lambda)$ comprises absorption by pure water, phytoplankton pigments, hydrosol particles, as well as by other particulates and colored dissolved organic matter, $\beta_{\mathrm{vrs}}(\lambda)$ is the total vibrational Raman scattering coefficient, which describes the energy transport out of the observation wavelength $\lambda$ to longer wavelengths, $N_{\mathrm{f}}$ is the number of fluorescing components, and the last term in the expression for $e_{2}(\lambda)$ is a fluorescence absorption part. Details about inherent optical properties (IOPs) of sea water and water constituents implemented in the current version of SCIATRAN can be found in [6]. As for atmospheric optical parameters, the IOPs can depend on the depth. However, for simplicity reasons we do not indicate this dependence explicitly.

The inelastic scattering source function $\mathcal{J}_{2}(\tau, \Omega, \lambda)$ in Eq. (14) is given following Haltrin and Kattawar [28] as:

$\mathcal{J}_{2}(\tau, \Omega, \lambda)=\frac{\omega_{2}(\lambda)}{4 \pi} \int_{\lambda^{\prime}<\lambda} \int_{\Omega} \rho_{2}^{\text {in }}\left(\Omega, \Omega^{\prime} ; \lambda^{\prime}, \lambda\right) I_{2}\left(\tau, \Omega^{\prime}, \lambda^{\prime}\right) d \Omega^{\prime} d \lambda^{\prime}$,

where we have introduced a spatial-frequency phase function of inelastic processes in the ocean as

$\mathcal{P}_{2}^{\text {in }}\left(\Omega, \Omega^{\prime} ; \lambda^{\prime}, \lambda\right)=\frac{r_{\mathrm{v}}\left(\lambda^{\prime}, \lambda\right) \mathcal{P}_{\mathrm{vrs}}\left(\Omega, \Omega^{\prime}\right)+\sum_{\mathrm{f}=1}^{N_{\mathrm{f}}} \sigma_{\mathrm{f}}\left(\lambda^{\prime}, \lambda\right) \mathcal{P}_{\mathrm{f}}\left(\Omega, \Omega^{\prime}\right)}{\beta_{2}(\lambda)}$,

Here $_{\mathrm{v}}\left(\lambda^{\prime}, \lambda\right)$ and $\mathcal{P}_{\mathrm{vrs}}\left(\Omega, \Omega^{\prime}\right)$ are vibrational Raman scattering coefficient and phase function, respectively, following [28] $\sigma_{\mathrm{f}}\left(\lambda^{\prime}, \lambda\right)$ will be referred to as the differential emission coefficient for fluorescence. Phase functions for all fluorescence components, $\mathcal{P}_{\mathrm{f}}\left(\Omega, \Omega^{\prime}\right)$, are omitted assuming isotropy of the fluorescence emission.

The implementation details of the vibrational Raman scattering, chl- $a$ and CDOM fluorescence are given in Appendices A, B.1, B.2, respectively.

\section{Solution of RTE including inelastic processes}

The approach to solve the BVP formulated above starts with splitting the total radiation field into a direct and diffuse components. The detailed description of this transformation in the case of coupled ocean-atmosphere model is given, e.g., by Blum et al. [6]. Furthermore, we assume that the rotational Raman scattering in the atmosphere and the vibrational Raman scattering, CDOM and chl- $a$ fluorescence in the ocean are minor effects and can be treated as linear perturbations $[28,80,81,85]$. The solution of the BVP in the case of coupled ocean-atmosphere system is performed using an iterative approach $[6,61]$. First, BVP for the atmosphere is solved using an a priori estimate for the water-leaving radiation (second term in the right-hand side of Eq. (6)) which is considered as the lower boundary condition for the atmosphere. The radiative field at the lower boundary of the atmosphere obtained in this manner is used then to formulate the upper boundary condition for the ocean in accordance with Eq. (6). Subsequently, BVP for the ocean is solved providing an updated lower boundary condition for the atmosphere and BVP for the atmosphere is solved then again. The iterative process is run until the results for the water-leaving radiation and 
the radiation entering the ocean converge, i.e., differences between the results from two subsequent iterations are below a pre-selected threshold.

According to this approach we formulate following two BVPs that need to be solved to obtain the radiation field in the coupled ocean-atmosphere system. The diffuse radiation field in the atmosphere is obtained solving iteratively the following BVP:

$\mu \frac{\partial I_{1}^{n}(\tau, \Omega, \lambda)}{\partial \tau}=-I_{1}^{n}(\tau, \Omega, \lambda)+J_{1}^{n}(\tau, \Omega, \lambda)+Q_{1}(\tau, \Omega, \lambda)$,

$I_{1}^{n}(0, \Omega, \lambda)=0, \quad \mu>0$,

$I_{1}^{\mathrm{n}}\left(\tau_{a}, \Omega, \lambda\right)=R_{\mathrm{a}}\left(\Omega, \Omega_{0}\right) F_{0}(\lambda) \mu_{0} e^{-\tau_{a} / \mu_{0}}$

$$
+\mathcal{R}_{\mathrm{a}} I_{1}^{n}\left(\tau_{a}, \Omega^{\prime}, \lambda\right)+\mathcal{T}_{\mathrm{wa}} I_{2}^{n-1}\left(\tau_{a}, \Omega^{\prime}, \lambda\right), \quad \mu<0,
$$

where the superscript $n=\{1,3,5, \ldots\}$ denotes the iteration number, the upper boundary condition, Eq. (21), manifests that there is no incoming diffuse radiation at the top of atmosphere, the first term in the lower boundary condition given by Eq. (22) describes the direct solar radiation reflected from the air-water interface, $I_{2}^{0}\left(\tau_{a}, \Omega^{\prime}, \lambda\right)$ in the right-hand side of this equation is set to zero in the current version of SCIATRAN, i.e., there is no waterleaving radiation at the first iteration in the atmosphere. The source functions for the diffuse radiation are defined as

$J_{1}^{n}(\tau, \Omega, \lambda)=\frac{\omega_{1}(\lambda)}{4 \pi} \int_{\Omega} \mathcal{P}_{1}\left(\Omega, \Omega^{\prime}\right) I_{1}^{n}\left(\tau, \Omega^{\prime}, \lambda\right) d \Omega^{\prime}$,

$$
\begin{aligned}
Q_{1}(\tau, \Omega, \lambda)= & \frac{\omega_{1}(\lambda)}{4 \pi}\left[\sum_{j=1}^{L} \int_{\Omega} \mathcal{P}_{1}^{\mathrm{in}}\left(\Omega, \Omega^{\prime} ; \lambda, \lambda_{j}\right) \mathcal{I}_{1}\left(\Omega^{\prime}, \lambda_{j}\right) d \Omega^{\prime}\right. \\
& \left.+\sum_{j=1}^{L} \mathcal{P}_{1}^{\mathrm{in}}\left(\Omega, \Omega_{0} ; \lambda, \lambda_{j}\right) F_{1}\left(\lambda_{j}\right)+\mathcal{P}_{1}\left(\Omega, \Omega_{0}\right) F_{1}(\lambda)\right],
\end{aligned}
$$

$F_{1}(\lambda)=F_{0}(\lambda) e^{-\tau / \mu_{0}}, \quad F_{1}\left(\lambda_{j}\right)=F_{0}\left(\lambda_{J}\right) e^{-\tau_{j} / \mu_{0}}$

where $\mathcal{I}_{1}\left(\Omega^{\prime}, \lambda_{j}\right)$ is the intensity of radiation field calculated at all wavelengths $\lambda_{j}$ accounting for coupling effects but excluding inelastic radiative processes, $\tau$ and $\tau_{j}$ are optical depths at wavelengths $\lambda$ and $\lambda_{j}$, respectively. The first term in the expression for the source function $Q_{1}(\tau, \Omega, \lambda)$ describes the multiple scattering in the spatial domain of the radiation scattered once in the frequency domain, the second describes the single scattering in the spatial domain of the radiation scattered once in the frequency domain, and the last term describes the contribution of the single elastic scattering from all relevant processes.

We note that expressions for the lower boundary condition and single scattering source function (Eqs. (22) and (24), respectively) are given for the wind-roughened air-water interface and planeparallel atmosphere. In the case of an absolutely flat surface the first term in Eq. (22) is omitted and an additional term describing the single scattering of the direct solar radiation reflected at the surface is introduced in Eq. (24) for the single scattering source function (see [6] for details). In the pseudo-spherical approximation a spherical shell atmosphere is assumed when calculating the attenuation of the direct solar light, which contributes into the lower boundary condition and single scattering source function, see Eqs. (22) and (25).

The diffuse radiation field in the ocean is obtained solving the following BVP: $\mu \frac{\partial I_{2}^{n}(\tau, \Omega, \lambda)}{\partial \tau}=-I_{2}^{n}(\tau, \Omega, \lambda)+J_{2}^{n}(\tau, \Omega, \lambda)+Q_{2}(\tau, \Omega, \lambda)$,

$I_{2}^{n}(0, \Omega, \lambda)=\mathcal{R}_{\mathrm{w}} I_{2}^{n}\left(\tau_{a}, \Omega^{\prime}, \lambda\right)+\mathcal{T}_{\mathrm{aw}} I_{1}^{n-1}\left(\tau_{a}, \Omega^{\prime}, \lambda\right), \quad \mu>0$,

$I_{2}^{n}\left(\tau_{0}, \Omega, \lambda\right)=\frac{1}{\pi} \int_{\Omega^{+}} R_{2}\left(\Omega, \Omega^{\prime}\right) I_{2}^{n}\left(\tau_{0}, \Omega^{\prime}, \lambda\right) \mu^{\prime} d \Omega^{\prime}, \quad \mu<0$,

where $n=\{2,4,6, \ldots\}$ denotes the iteration number. Source functions for the diffuse radiation are defined as

$J_{2}^{n}(\tau, \Omega, \lambda)=\frac{\omega_{2}(\lambda)}{4 \pi} \int_{4 \pi} \mathcal{P}_{2}\left(\Omega, \Omega^{\prime}\right) I_{2}^{n}\left(\tau, \Omega^{\prime}, \lambda\right) d \Omega^{\prime}$,

$$
\begin{aligned}
Q_{2}(\tau, \Omega, \lambda)= & \frac{\omega_{2}(\lambda)}{4 \pi}\left[\int_{\lambda^{\prime}<\lambda} \int_{4 \pi} \mathcal{P}_{2}^{\mathrm{in}}\left(\Omega, \Omega^{\prime} ; \lambda^{\prime}, \lambda\right) \mathcal{I}_{2}\left(\tau, \Omega^{\prime}, \lambda^{\prime}\right) d \Omega^{\prime} d \lambda^{\prime}\right. \\
& \left.+\int_{\lambda^{\prime}<\lambda} \mathcal{P}_{2}^{\mathrm{in}}\left(\Omega, \Omega_{0} ; \lambda^{\prime}, \lambda\right) F_{2}\left(\lambda^{\prime}\right) d \lambda^{\prime}+\mathcal{P}_{2}\left(\Omega, \Omega_{0}\right) F_{2}(\lambda)\right],
\end{aligned}
$$

$F_{2}(\lambda)=F_{0}(\lambda) \frac{\mu_{0}}{\mu_{0}^{\prime}} T_{\mathrm{F}}\left(\mu_{0}\right) e^{-\tau_{a} / \mu_{0}} e^{-\tau / \mu^{\prime} 0}$.

Here, $I_{2}\left(\tau, \Omega^{\prime}, \lambda^{\prime}\right)$ is the intensity of radiation field in the ocean calculated at all wavelengths $\lambda^{\prime}<\lambda$ accounting for coupling effects but excluding all inelastic processes, $T_{\mathrm{F}}\left(\mu_{0}\right)$ is the Fresnel transmission coefficient of the air-water interface, $\tau$ is the optical depth counted from the surface ocean, and $\mu_{0}^{\prime}$ is the cosine of the solar angle in the ocean defined according to the Snell law [7] as $\mu_{0}^{\prime}=\sqrt{1-\left(1-\mu_{0}^{2}\right) / n^{2}}$, where $n$ is the real part of the water refractive index. We assume throughout this paper that the refractive index of the air is equal to 1 . The multiplier $\mu_{0} / \mu_{0}^{\prime}$ is introduced in the expression for $F_{2}(\lambda)$ to ensure the energy conservation of the direct solar radiation just above and just below the ocean surface.

The first and second terms in the expression for the source function $Q_{2}(\tau, \Omega, \lambda)$ describe the multiple and single scattering, respectively, in the spatial domain of the radiation scattered once in the frequency domain. The last term describes the elastic single scattering by all relevant processes. It is worth to notice that transfer of the direct solar radiation through the ocean is modeled neglecting the wind-roughness of the water surface, i.e., a flat air-water interface is assumed. The discussion of this approximation can be found in Fell and Fischer [20]. In the case of pseudo-spherical approximation the solar attenuation term $e^{-\tau_{a} / \mu_{0}}$ in Eq. (31) is replaced by the spherical transmission function and $\mu_{0}$ is calculated accounting for the sphericity of the atmosphere.

To solve the above formulated BVPs we employ the Fourier analysis to separate the zenith and azimuthal dependence of the intensity and the discrete-ordinates technique $[12,67,72,78]$ for the reduction of integro-differential equations to the system of ordinary differential equations. In particular, the expansion of the intensity and phase functions into Fourier series allows the BVP to be solved for each Fourier harmonic of the intensity independently which significantly reduces the total dimension of the problem. According to the discrete-ordinates technique, the radiation field is represented by $N$ upwelling and $N$ downwelling streams, producing the intensity pairs $I\left(\tau, \pm \mu_{i}\right)$ in the discrete-directions $\pm \mu_{i}$, where $\mu_{i}$ are quadrature points of the double-Gauss scheme (see, e.g., [78] for details) adopted in SCIATRAN. 
To describe the radiation field in water we have implemented the so-called coupled underwater quadrature points method as suggested by Jin et al. [32]. The detailed discussion of this method is given, e.g., by He et al. [31].

Calculations of definite integrals over the wavelength in Eq. (30) are performed using the trapezoidal rule with uniform grid. The grid spacing is an input parameter. Having defined the Gaussian-quadrature points and applying a quadrature formula to replace integrals over the direction cosine by finite sums in the radiative transfer equation, one arrives for the $m$-th Fourier harmonic of intensity at a system of coupled first order ordinary linear differential equations in the optical depth $\tau$ :

$\left[ \pm \mu_{i} \frac{d}{d \tau}+1\right] I_{s}^{m}\left(\tau, \pm \mu_{i}\right)=J_{s}^{m}\left(\tau, \pm \mu_{i}\right)+Q_{s}^{m}\left(\tau, \pm \mu_{i}\right), \quad s=1,2$,

where we have omitted the superscript $n$ for the iteration number. In the following discussion we do not denote explicitly that the Gaussian quadrature points and weights in the atmosphere and ocean are different. The discrete-ordinates representation of the source function is given by

$$
\begin{aligned}
J_{s}^{m}\left(\tau, \pm \mu_{i}\right)= & \frac{\omega}{2} \sum_{j=1}^{N_{s}} w_{j} \sum_{l=m}^{K_{s}} \beta_{s, l} P_{l}^{m}\left( \pm \mu_{i}\right) \\
& \times\left[P_{l}^{m}\left(\mu_{j}\right) I_{s}^{m}\left(\tau, \mu_{j}\right)+P_{l}^{m}\left(-\mu_{j}\right) I_{s}^{m}\left(\tau,-\mu_{j}\right)\right],
\end{aligned}
$$

where $N_{\mathrm{s}}$ and $w_{j}$ are the number of Gaussian quadrature points and their weights, respectively, $P_{l}^{m}(\mu)$ is the associated Legendre function, $P_{l}^{0}(\mu)=P_{l}(\mu)$ is the Legendre function, and $\beta_{\mathrm{s}, l}$ are expansion coefficients of the phase function in the Legendre series.

In the framework of the discrete-ordinates technique, solution of Eq. (32) can be obtained analytically in the case of a vertically homogeneous medium. To exploit this advantage we assume that both atmosphere and ocean consist of several vertically homogeneous layers. Within an $l$-th layer whose top and bottom are defined by the optical depth $\tau_{l-1}$ and $\tau_{l}$, respectively, the solution of RTE is given as a sum of the general solution of a homogeneous equation, i.e., setting $Q_{s}^{m}\left(\tau, \pm \mu_{i}\right)$ to zero in Eq. (32), and a particular solution of the inhomogeneous equation

$\mathbf{I}_{ \pm}(\tau)=\mathbf{I}_{ \pm}^{h}(\tau)+\mathbf{I}_{ \pm}^{p}(\tau)$,

where $\tau \in\left[\tau_{l-1}, \tau_{l}\right]$, superscripts $h$ and $p$ denote the general and particular solutions, respectively. We drop here and below the subscript $s$ because the solution of Eq. (32) is obtained in the same way in both atmosphere and ocean.

The vector $\mathbf{I}_{ \pm}(\tau)$ is given by

$\mathbf{I}_{ \pm}(\tau)=\left[I\left(\tau, \pm \mu_{1}\right), I\left(\tau, \pm \mu_{2}\right), \ldots, I\left(\tau, \pm \mu_{N}\right)\right]^{T}$,

and the superscript $T$ denotes the transposition. The analytical representation of the general solution of a homogeneous equation is given in the framework of the discrete-ordinates method as follows $[67,68]$ :

$\mathbf{I}_{ \pm}^{h}(\tau)=\sum_{j=1}^{N}\left[A_{j} \boldsymbol{\Phi}_{ \pm}\left(\nu_{j}\right) e^{-\left(\tau-\tau_{l-1}\right) / \nu_{j}}+B_{j} \boldsymbol{\Phi}_{\mp}\left(\nu_{j}\right) e^{-(\tau l-\tau) / \nu_{j}}\right]$,

where vectors $\boldsymbol{\Phi}_{ \pm}\left(\nu_{j}\right)$ and collection of separation constants $\left\{\nu_{j}\right\}$ are obtained solving the eigenvalue problem for the homogeneous equation (see [68] for details), $A_{j}$ and $B_{j}$ are arbitrary constants.

Among different techniques to derive a particular solution of an inhomogeneous equation (see, e.g., [56]) we have chosen a very flexible approach based on the infinite-medium Green functions $[1,67]$. An elegant and simple representation of infinite medium Green functions in the framework of discrete-ordinates technique was derived by Siewert [68]:

$\mathbf{G}_{ \pm}\left(\tau: x, \pm \mu_{\alpha}\right)=\sum_{j=1}^{N} \boldsymbol{\Phi}_{ \pm}\left(\nu_{j}\right) \tilde{A}_{j}\left( \pm \mu_{\alpha}\right) e^{-(\tau-x) / \nu_{j}}, \quad \tau>x$,

$\mathbf{G}_{ \pm}\left(\tau: x, \pm \mu_{\alpha}\right)=-\sum_{j=1}^{N} \boldsymbol{\Phi}_{\mp}\left(\nu_{j}\right) \tilde{B}_{j}\left( \pm \mu_{\alpha}\right) e^{-(x-\tau) / \nu_{j}}, \quad \tau<x$,

where $\mu_{\alpha}$ with $\alpha=1, \ldots, N$ are the Gaussian quadrature points, $\tilde{A}_{j}\left( \pm \mu_{\alpha}\right)$ and $\tilde{B}_{j}\left( \pm \mu_{\alpha}\right)$ are arbitrary constants, $x \in\left[\tau_{l-1}, \tau_{l}\right]$. The analytical expressions for constants $\tilde{A}_{j}\left( \pm \mu_{\alpha}\right)$ and $\tilde{B}_{j}\left( \pm \mu_{\alpha}\right)$ are given by Siewert. The general expression for a particular solution is obtained integrating the right-hand side of the inhomogeneous RTE with Green's functions, i.e.,

$$
\begin{aligned}
\mathbf{I}_{ \pm}^{p}(\tau)= & \sum_{\alpha=1}^{N} \int_{\tau_{l-1}}^{\tau_{l}}\left[\mathbf{G}_{ \pm}\left(\tau: x, \mu_{\alpha}\right) Q\left(x, \mu_{\alpha}\right)\right. \\
& \left.+\mathbf{G}_{ \pm}\left(\tau: x,-\mu_{\alpha}\right) Q\left(x,-\mu_{\alpha}\right)\right] d x .
\end{aligned}
$$

Substituting into this equation the analytical expressions for Green's functions given by Eqs. (37) and (38) and performing an integration with functions $Q_{1}(\tau, \Omega, \lambda)$ and $Q_{2}(\tau, \Omega, \lambda)$ given by Eqs. (24) and (30), respectively, we obtain all needed particular solutions for the atmosphere and ocean. The main details of derivation and analytical expressions for $\tau$-depending coefficients of particular solutions are similar to the case of rotational Raman scattering in the atmosphere and are given in [62].

The analytical solution of RTE is given within each homogeneous layer by Eq. (34). It contains $2 N$ arbitrary constants $\left\{A_{j}\right\}$ and $\left\{B_{j}\right\}$ according to Eq. (36). All constants are obtained employing the boundary conditions and requiring the continuity of the intensity across layer interfaces. From the mathematical point of view a linear algebraic system of equations with respect to $M \times 2 N$ constants needs to be solved, where $M$ is the number of layers in the atmosphere or ocean. Further details can be found in [61].

\section{Selected simulation results}

In this section we present selected results obtained with the SCIATRAN code. In particular, we consider the impact of oceanatmosphere coupling on the rotational Raman scattering (Section 4.1), filling-in of Fraunhofer lines by the vibrational Raman scattering in the coupled ocean-atmosphere system (Section 4.2), and impact of CDOM fluorescence on the spectra of the scattered solar light (Section 4.3).

\subsection{Rotational Raman scattering in a coupled ocean-atmosphere system}

A verification of RRS within the uncoupled SCIATRAN model and comparisons to results obtained previously [85] have been presented by Rozanov and Vountas [62]. Here we only consider the impact of coupling effects on the filling-in of Fraunhofer lines by the rotational Raman scattering.

The simulations were done for a spectral range of $410-450 \mathrm{~nm}$. The main reason for this choice is that spectral windows within this interval are routinely used in the framework of the DOAS technique to retrieve the vertical columns of $\mathrm{NO}_{2}$ [57] and iodine monoxide [65] from satellite measurements of the scattered solar light.

The Ring spectra investigated in this study were calculated according to the following equation: 
$r(\lambda ; \vartheta, \varphi)=\ln \frac{I^{+}(\lambda ; \vartheta, \varphi)}{I^{-}(\lambda ; \vartheta, \varphi)}$,

where $I^{+}(\lambda ; \vartheta, \varphi)$ and $I^{-}(\lambda ; \vartheta, \varphi)$ denote the intensity calculated including and excluding rotational Raman scattering, respectively. The calculations were performed in the Rayleigh-aerosol atmosphere accounting for gaseous absorption by $\mathrm{O}_{3}$ and $\mathrm{NO}_{2}$. The aerosol model was selected in accordance with the LOWTRAN aerosol parameterization [39] as follows:

- Boundary layer 0-2 km: maritime aerosol type, $50 \mathrm{~km}$ visibility, $80 \%$ humidity.

- Troposphere $2-10 \mathrm{~km}$ : $50 \mathrm{~km}$ visibility, $80 \%$ humidity.

- Stratosphere 10-30 km: moderate volcanic loading, background aerosol type.

- Mesosphere 30-60 km: normal mesosphere aerosol loading.

- These settings result in the aerosol optical thickness of $\sim 0.14$ at $550 \mathrm{~nm}$.

The refractive index of water was set to 1.34 and the air-water interface was assumed to be wind-roughed with the wind speed $5 \mathrm{~m} / \mathrm{s}$. The depth of the ocean was set to $500 \mathrm{~m}$ and a black bottom was assumed. The absorption and elastic-scattering coefficients of pure sea water were selected following Blum et al. [6]. The chlorophyll concentration of $0.1 \mathrm{mg} / \mathrm{m}^{3}$ was set between 0 and $40 \mathrm{~m}$ depth.

A high-resolved extraterrestrial solar spectrum reported by Kurucz [42] pre-convoluted with Gaussian slit function was used. The half width at half maximum of the slit function was set to $0.24 \mathrm{~nm}$ to match the spectral resolution of the SCIAMACHY instrument $[8,25]$.

The Ring spectra calculated according to Eq. (40) are presented in Fig. 1 for the satellite (upper left panel) and ground-based (upper right panel) observation geometries. By comparing upper left and upper right panels in Fig. 1 one can see that the effect of coupling is stronger for the satellite observation geometry (at least at the cloud-free conditions). To estimate the coupling effect quantitatively, let us introduce the differential Ring spectrum obtained by subtracting a polynomial from the Ring spectrum, i.e.,

$r_{d}(\lambda)=r(\lambda)-\sum_{i=0}^{N} a_{i} \lambda^{i}$,

and define the scaling factor $c$ obtained by fitting the Ring spectrum simulated neglecting the coupling effect, $r_{d}{ }^{e}$, to that obtained accounting for the coupling:

$\left\|r_{d}(\lambda)-\operatorname{cr}_{d}^{e}(\lambda)\right\|^{2} \rightarrow \min$.

Setting the polynomial order, $N$, to 3 in Eq. (41), the scaling factors of 1.048 and 1.025 were obtained for the satellite and groundbased observation geometry, respectively. The middle panels of Fig. 1 show the differential Ring spectra $r_{d}(\lambda)$ and $c r_{d}^{e}(\lambda)$ for both considered observation geometries. It follows that for satellite observation geometry the filling-in of Fraunhofer lines is underestimated by $\sim 5 \%$ when the coupling effects are ignored. This might be crucial if absolute values of filling-in are used to estimate atmospheric parameters as, e.g., in the case of aerosol retrieval $[87,88]$.

However, if the Ring spectrum is used in the framework of the DOAS technique [58], the coupling effects are of minor importance because the residual between the differential Ring spectrum $r_{d}(\lambda)$ obtained accounting for the coupling effects and scaled differential Ring spectrum calculated neglecting the coupling $\operatorname{cr}_{d}^{e}(\lambda)$ does not exceed $\sim 3 \cdot 10^{-5}$ (see lower panels of Fig. 1 ). This means that the coupling effect is mostly compensated by the scaling factor.
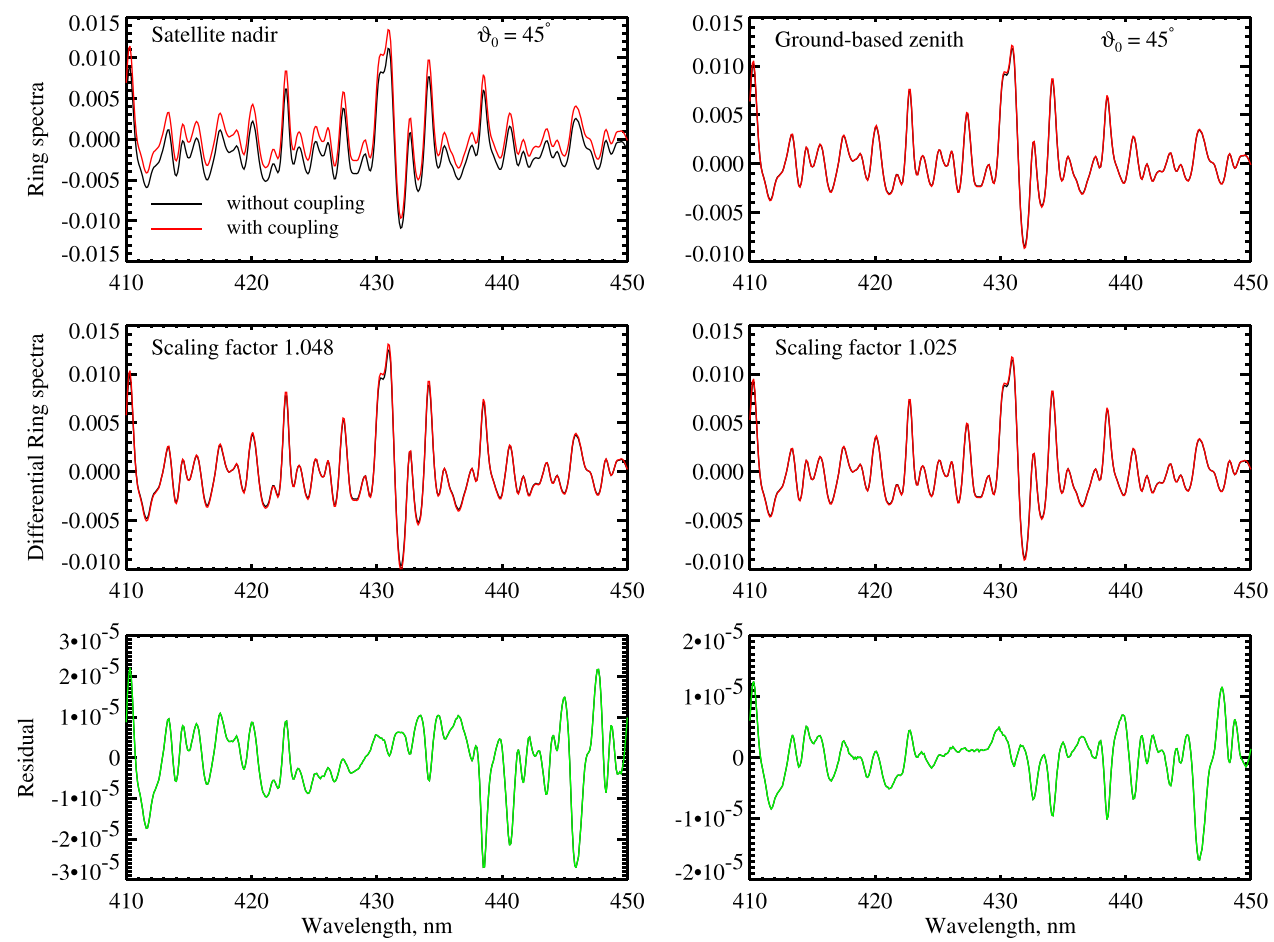

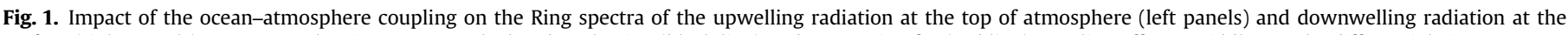

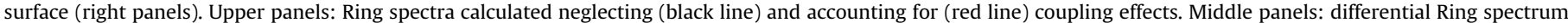

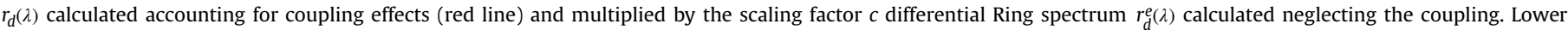
panels: difference $r_{d}(\lambda)-c r_{d}^{e}(\lambda)$. (For interpretation of the references to color in this figure caption, the reader is referred to the web version of this paper.) 


\subsection{Vibrational Raman scattering in a coupled ocean-atmosphere system}

\subsubsection{Filling-in of Fraunhofer lines by VRS}

In this section, we consider the filling-in of Fraunhofer lines in the atmosphere and ocean by vibrational Raman scattering. The filling-in of Fraunhofer lines is calculated using the ratio of radiance in the core and wing of a Fraunhofer line. Following Kattawar and $\mathrm{Xu}$ [37], we introduce this ratio as

$f_{I}=\frac{I_{\mathrm{c}}+I_{\text {in }}}{I_{\mathrm{w}}+I_{\mathrm{in}}}$,

where $I_{\mathrm{c}}$ and $I_{\mathrm{w}}$ are elastic radiances in the core and wing, respectively, and $I_{\text {in }}$ is a contribution of inelastically scattered radiation.

As pointed out in [37] Eq. (43) assumes that a Fraunhofer line is significantly narrower than the vibrational Raman band. Therefore, the same value of $I_{\text {in }}$ in the core and in the wing of the solar Fraunhofer line can be used. This is a reasonable assumption, e.g., for the $H_{\beta}$ Fraunhofer line considered below, because the full width at half maximum of the VRS band is about $8 \mathrm{~nm}$ while that of the $H_{\beta}$ Fraunhofer line is about $0.06 \mathrm{~nm}$ [37].

Calculations of the core-to-wing ratio were performed setting the atmospheric and oceanic parameters in accordance with [37]:

- The atmosphere was assumed to have an optical depth of 0.15 and to be free of aerosol, i.e. only Rayleigh scattering was considered.

- The refractive index of water was set to 1.34 and the air-water interface was assumed flat.

- The absorption, elastic-scattering, and total Raman scattering coefficients of pure sea water, $a_{w}, b_{w}$, and $b_{r}$, respectively, were set at excitation $(417 \mathrm{~nm})$ and emission $(486 \mathrm{~nm})$ wavelengths to

$a_{w}(417)=0.0156 \mathrm{~m}^{-1}, \quad a_{w}(486)=0.0188 \mathrm{~m}^{-1}$,

$b_{w}(417)=0.0064 \mathrm{~m}^{-1}, \quad b_{w}(486)=0.0032 \mathrm{~m}^{-1}$,

$b_{r}(417)=0.00264 \mathrm{~m}^{-1}, \quad b_{r}(486)=0.00143 \mathrm{~m}^{-1}$.

The change of the inherent optical properties across the width of the Raman band was neglected. Note that the large Raman scenario (LRam) as presented in [37] was used.

- The depth of the ocean was set to $1000 \mathrm{~m}$ and a black bottom was assumed.

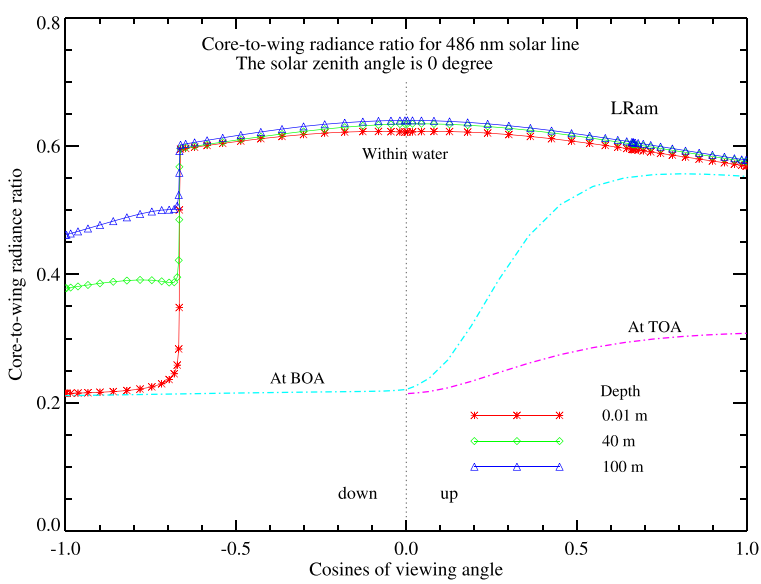

Fig. 2. Core-to-wing ratio for the downwelling and upwelling radiances, $f_{I}$, for the $\mathrm{H}_{\beta}$ Fraunhofer line as a function of viewing angle for three water depths, the top of atmosphere and the bottom of atmosphere, denoted in figure as "Within water", "At TOA", and "At BOA", respectively. The solar zenith angle is set to $0^{\circ}$. Negative and positive cosines of viewing angles at $x$-axis denote downward and upward traveling radiation, respectively. (For interpretation of the references to color in this figure caption, the reader is referred to the web version of this paper.)
- Following Beckers et al. [3], the solar irradiance was set to 132 and $646 \mathrm{~W} \mathrm{~m}^{-2} \mu \mathrm{m}^{-1}$ at the core and wing of $H_{\beta}$ Fraunhofer line, respectively.

Fig. 2 shows the radiance ratio, $f_{I}$, as a function of viewing angle at three different depths in the ocean, just above the ocean surface, and at the top of atmosphere. In the following the results shown in Fig. 2 will be discussed separately for the atmosphere and ocean.

Atmosphere: The filling-in of Fraunhofer lines by the vibrational Raman scattering occurred within the ocean affects the radiation traveling through the atmosphere. The core-to-wing radiance ratios in the atmosphere are shown in Fig. 2 by dash-dotted lines. For the downwelling radiation near the surface (negative cosines of viewing angles) the filling-in is very weak and almost independent of the viewing angle (see respective $f_{I}$ values in Fig. 2 marked "At BOA"). This can be explained by the fact that only a small part of the inelastic radiation transmitted from the ocean into the atmosphere is scattered back to the ocean surface. (Note: In this scenario the optical thickness of the Rayleigh atmosphere was set to 0.15.) In contrast, a strong dependence of the solar Fraunhofer line filling-in on the viewing angle is observed for the upwelling radiation near the air-water interface (positive cosines of viewing angles). In particular, $f_{I}$ increases from $\sim 0.22$ near the horizon to $\sim 0.55$ at zenith because the inelastic radiation is transmitted from the ocean into the atmosphere within the Fresnel cone and the transmission coefficient increases with decreasing incident angle.

For the upwelling radiation at the top of atmosphere (Fig. 2 marked "At TOA"), the viewing angle dependence of the filling-in of solar Fraunhofer lines is similar to that at the bottom of the atmosphere, i.e., $f_{I}$ increases with decreasing viewing angle. However, in this case the filling-in towards the zenith direction $(\cos \vartheta=1)$ is significantly smaller as compared to the bottom of atmosphere because the contribution of the elastically scattered radiation is more significant.

Ocean: The core-to-wing radiance ratios in the ocean are shown in Fig. 2 by solid lines with symbols. It can be seen that the radiance ratio shows a large jump for the downwelling radiation at the critical angle of the flat ocean surface $\left(\cos \vartheta_{c}=-0.67\right)$. Viewing angles less than $\vartheta_{c}=48.6^{\circ}$ (from the downward direction) are within the so-called Fresnel cone where the upwelling radiation in the ocean is transmitted into the atmosphere. Viewing angles greater than $\vartheta_{c}$ are within the so-called total reflection region where the upwelling radiation in the ocean is totally internally reflected back into the ocean. Fig. 2 demonstrates a very strong decrease of the downwelling radiation filling-in within the Fresnel cone. We note that results for the filling-in of the $H_{\beta}$ Fraunhofer line presented in Fig. 2 are in line with those from Kattawar and Xu (see Fig. 6 right-upper panel in [37]) demonstrating that all significant characteristics of filling-in in the ocean are well reproduced by SCIATRAN. The selected quantitative comparisons with results from Kattawar and Xu are given in Section 5.2.

In order to explain the results presented above, let us rewrite Eq. (43) in a form which is more convenient for theoretical analysis. In particular, we introduce the core-to-wing ratio of a Fraunhofer solar line without a contribution of inelastic scattering as

$D_{0}=\frac{I_{\mathrm{c}}}{I_{\mathrm{w}}}$.

Dividing the nominator and denominator of Eq. (43) by $I_{\mathrm{W}}$ and using Eq. (44), we obtain

$f_{I}=\frac{D_{0}+r_{\text {in }}}{1+r_{\text {in }}}$ 
where $r_{\text {in }}=I_{\text {in }} / I_{\mathrm{w}}$ denotes the ratio of the inelastic to elastic radiance in the wing of the solar Fraunhofer line.

The elastic and inelastic downward radiances as well as their ratios were simulated setting the extraterrestrial solar flux to the fixed values of $1 \mathrm{~W} \mathrm{~m}^{-2} \mathrm{~nm}^{-1}$ in the wing and to $0.2 \mathrm{~W} \mathrm{~m}^{-2} \mathrm{~nm}^{-1}$ in the core of the solar Fraunhofer line. The dependence of $I_{w}, I_{\mathrm{in}}$, and $r_{\text {in }}$ on the viewing angle is presented in Fig. 3.

Near the air-water interface $(0.01 \mathrm{~m})$ we observe an increase of the elastic and a strong decrease of the inelastic component of the downwelling radiation within the Fresnel cone as compared to their respective values outside of the Fresnel cone. The former is caused by a significant contribution of radiation which is transmitted from the atmosphere into ocean within the Fresnel cone while the latter results from a significant transmission of the upwelling inelastic radiation from the ocean into the atmosphere and a small reflection back into the ocean. The impact of the air-water interface decreases with increasing depth. As a result, the difference between the inelastic component of the downwelling radiation within and outside of the Fresnel cone decreases with increasing depth. In the considered scenario the ratio $r_{\text {in }}$ (Fig. 3 , right panel) strongly depends on the depth within the Fresnel cone demonstrating a significant increase of inelastic scattering with depth.

Outside the Fresnel cone, within the region of the total internal reflection, contributions of the elastic and inelastic components in the downwelling radiation are similar. It follows from the right panel of Fig. 3 that the ratio $r_{\text {in }}$ is nearly 1 within the region of the total reflection. Substituting the ratio $r_{\text {in }}$ equal to 1 into Eq. (45) and accounting for that $D_{0}=0.2$, we have in the region of the total internal reflection $f_{I}=0.6$. This demonstrates that the core-to-wing ratio $\left(f_{I}\right)$ for the downwelling radiance presented in Fig. 2 is explained due to the fact that contributions of the elastic and inelastic components are similar.

\subsubsection{Broadband spectral structure caused by VRS}

The broadband spectral structure resulted from VRS is considered in this section. For this purpose the absolute VRS signal,
$\Delta(\lambda)$, is introduced as

$\Delta(\lambda)=I^{+}(\lambda)-I^{-}(\lambda)$,

where $I^{+}(\lambda)$ and $I^{-}(\lambda)$ are the TOA radiances calculated including and excluding VRS within the ocean. The difference $\Delta(\lambda)$ describes the net contribution of VRS to the TOA radiance.

Generally, the spectral behavior of the VRS signal depends on the absorption of radiation in the atmosphere and ocean, on the spectral dependence of the vibrational Raman scattering coefficient, and on the structure of the extraterrestrial solar spectrum. The middle panel of Fig. 4 shows VRS spectra calculated using two different (realistic and constant) solar irradiance spectra (Fig. 4, upper panel). The calculations were performed for the same scenario as described in Section 4.1 but with the spectral sampling of $5 \mathrm{~nm}$. The reduced sampling was used to better demonstrate the broadband spectral structure. The observation and illumination geometry corresponds to the nadir viewing instrument and the overhead sun.

To analyze the VRS signal with respect to solar spectral structures, $\Delta(\lambda)$ was calculated first using an artificial constant solar spectrum in the model run, and then with a realistic extraterrestrial solar spectrum (see Section 4.1). For the constant solar spectrum (Fig. 4, middle panel), $\Delta(\lambda)$ has a smooth unstructured dependence on the wavelength with a broad maximum in the spectral range of $420-470 \mathrm{~nm}$. The decrease of $\Delta(\lambda)$ to shorter wavelengths is caused by the ozone absorption in the atmosphere, whereas the decrease to longer wavelengths is due to the liquid water absorption in the ocean.

When using a realistic extraterrestrial solar spectrum (Fig. 4, upper panel), $\Delta(\lambda)$ reproduces its spectral features because of the trans-spectral nature of the VRS process. In particular, the jump in the VRS signal at $460-470 \mathrm{~nm}$ is associated with the corresponding jump of solar irradiance in the spectral range 390-400 nm caused by the absorption in Ca II ( $\mathrm{H}$ and $\mathrm{K}$ ) Fraunhofer lines.

To illustrate the spectral redistribution of solar energy caused by the VRS process we have selected two emission wavelengths at $410 \mathrm{~nm}$ and $480 \mathrm{~nm}$ located at the local minimum and maximum
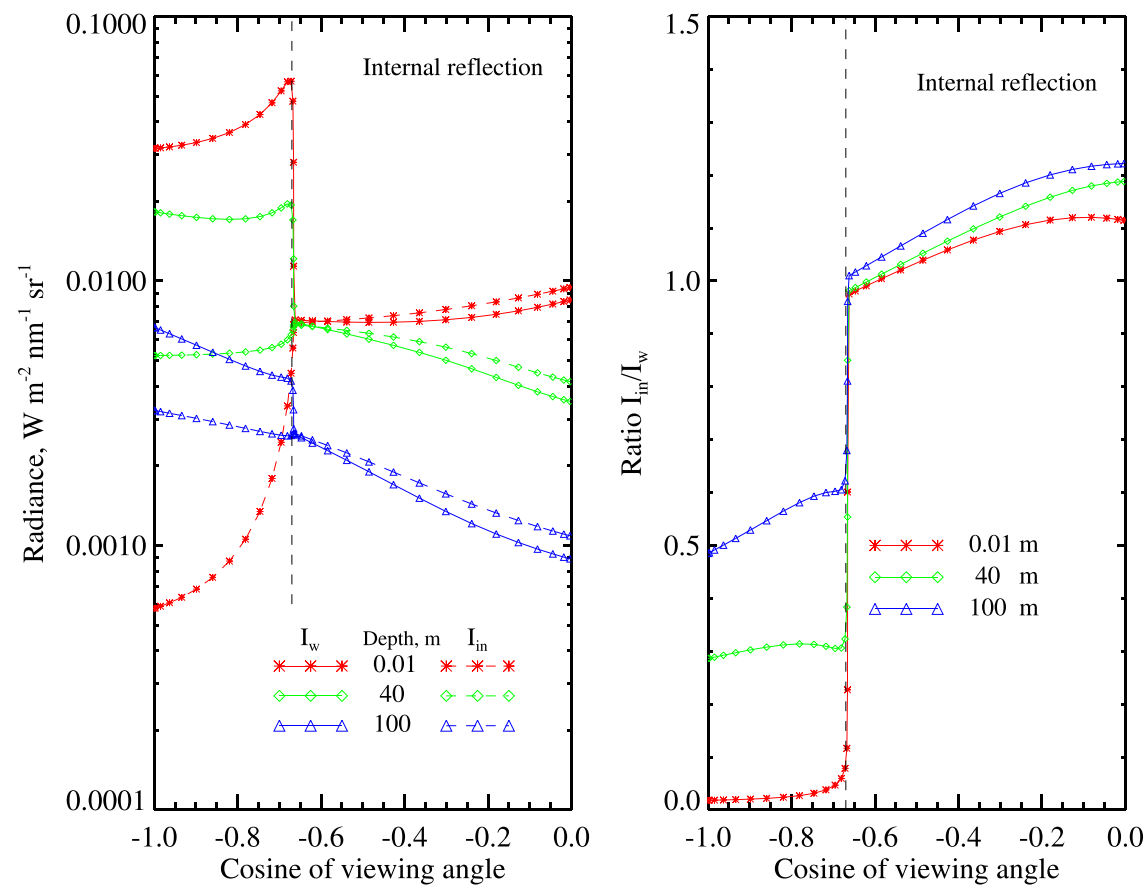

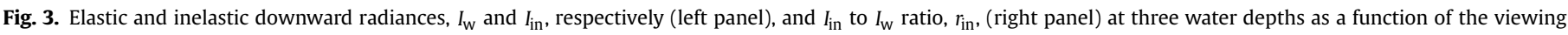

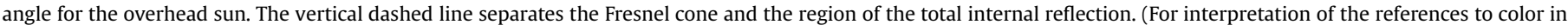
this figure caption, the reader is referred to the web version of this paper.) 

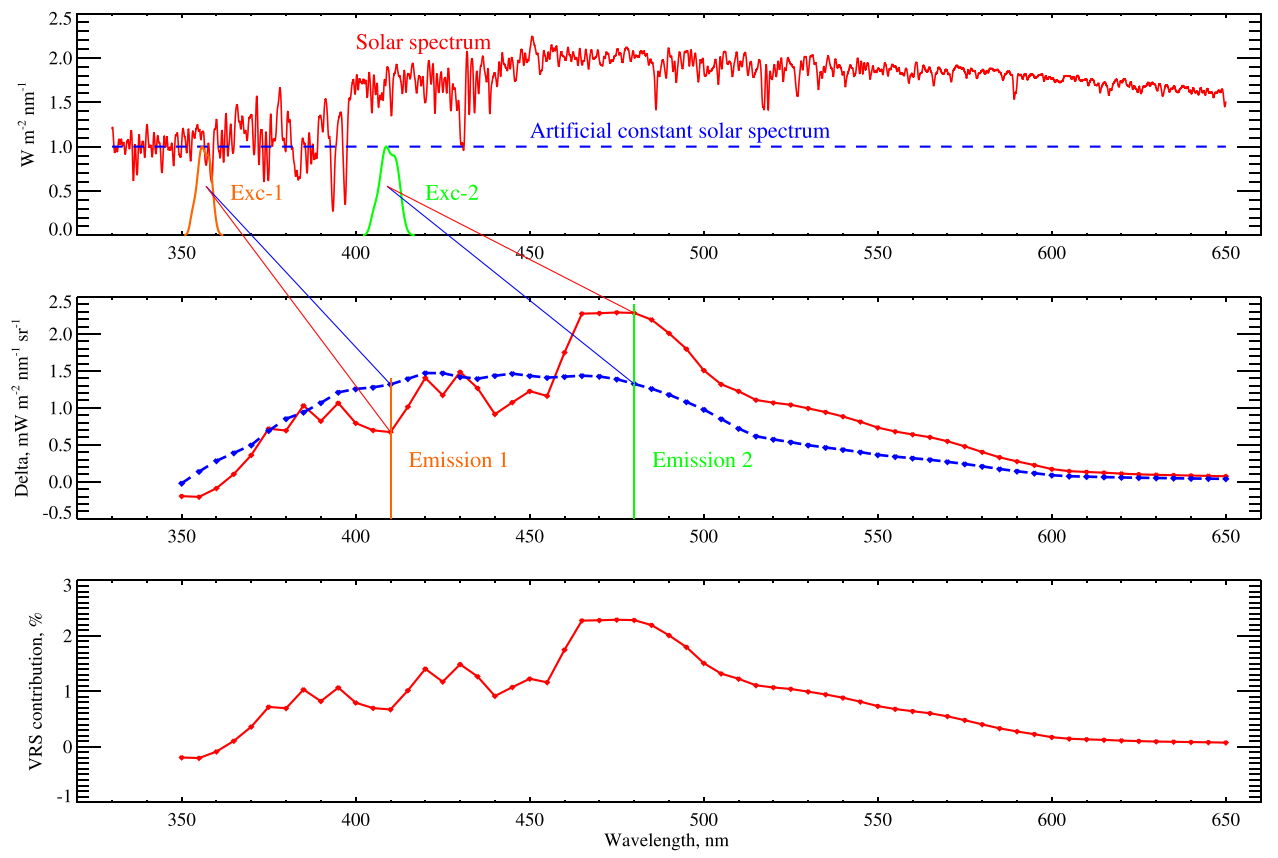

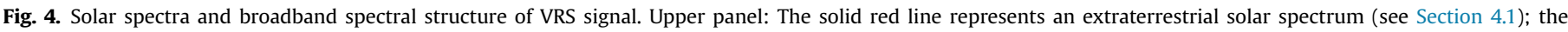

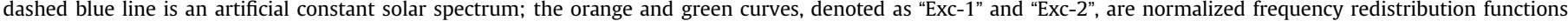

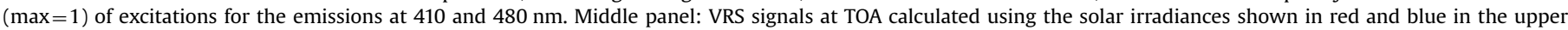

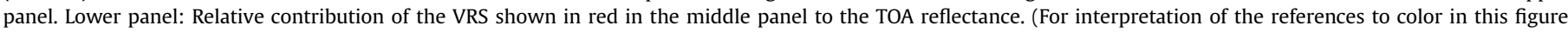
caption, the reader is referred to the web version of this paper.)

of the VRS signal (see Fig. 4). The corresponding excitation spectral ranges calculated using expression (A.2) for the frequency redistribution function of Raman scattered light are depicted in the upper panel of Fig. 4 by orange and green curves denoted as "Exc1 " and "Exc-2". By comparing positions of excitation ranges and emission wavelengths, it is obvious that the characteristic jump of the VRS spectrum at $460-470 \mathrm{~nm}$ is induced by the equivalent jump in the solar spectrum at 390-400 nm. This feature in the VRS signal was also identified by Peters et al. [54] and Dinter et al. [17] who retrieved the VRS signal in water from ship-borne and satellite measurements of the scattered solar radiation, respectively (see Sections 6.2 and 6.3).

The relative contribution of VRS to the TOA radiance shown in the lower panel of Fig. 4 does not exceed $~ 3 \%$ in the case under consideration.

\subsection{Filling-in and broadband spectral structure by the CDOM fluorescence}

Fluorescent dissolved organic matter, a fluorescent subgroup of CDOM, is a complex mixture of many organic molecules. Usually two major types of fluorophores are separated: humic-like, and protein-like (or amino acid-like). The former shows a broader peak with emission wavelengths typically longer than $400 \mathrm{~nm}$, whereas the latter displays narrower peaks with emission wavelengths shorter than $400 \mathrm{~nm}[14]$.

Two approaches to model CDOM fluorescence, which account for more than one CDOM component, were implemented in SCIATRAN:

- Fulvic and Humic Acids (FHA) approach based on the exponential approximation of the fluorescence efficiency function suggested by Hawes et al. [30] (see Appendix B.2.1).

- Additive Fluorescent Components (AFC) approach based on PARAllel FACtor (PARAFAC) analysis which is widely applied to excitation-emission matrix fluorescence spectroscopy measurements of CDOM fluorescence [76] (see Appendix B.2.2).
The filling-in of Fraunhofer lines by CDOM fluorescence was investigated using the FHA approach. The core-to-wing ratio for the $H_{\beta}$ Fraunhofer line near $486 \mathrm{~nm}$ was calculated according to Eq. (43) for the upwelling radiation at three observation levels: at the top and bottom of the atmosphere as well as just below the ocean surface. The calculations were performed with SCIATRAN in an aerosol-free atmosphere accounting for the molecular scattering and gaseous absorption by $\mathrm{O}_{3}$ and $\mathrm{NO}_{2}$. In addition to the absorption of liquid water, only absorption and fluorescence of CDOM were included in the ocean. Concentrations of both fulvic and humic acids were set to $1 \mathrm{~g} \mathrm{~m}^{-3}$. In order to compare the filling-in by CDOM and by VRS presented in Section 4.2.1 a highresolved extraterrestrial solar spectrum reported by Kurucz [42] was pre-convolved with Gaussian slit function. The half width at half maximum of the slit function was selected to match the spectral resolution of the solar spectrum given by Beckers et al. [3] which was used in Section 4.2.1.

The radiance ratio, $f_{I}$ (see Eq. (43)), as a function of viewing angle at the top and at the bottom of atmosphere as well as just below the ocean surface, is shown in Fig. 5. It follows that the filling-in of the $H_{\beta}$ Fraunhofer line varies with the viewing angle in a similar manner as the filling-in caused by VRS (see Fig. 2). In particular, $f_{I}$ increases at TOA and BOA with decreasing viewing angle and shows opposite dependence just below the ocean surface. However, for the selected scenario the filling-in by CDOM fluorescence is significantly weaker than the VRS signal for clear water. The dependence of the filling-in on the viewing angle is governed by the contribution of the elastically scattered radiation, which is stronger for high zenith angles (low cosines) and at the top of atmosphere. In addition, inelastically scattered radiation is transmitted from the ocean into the atmosphere more efficiently for low incident angles. The upwelling fluorescence signal just below the ocean surface does not vary significantly with the viewing angle, and the filling-in effect is only slightly stronger for large viewing angles. 


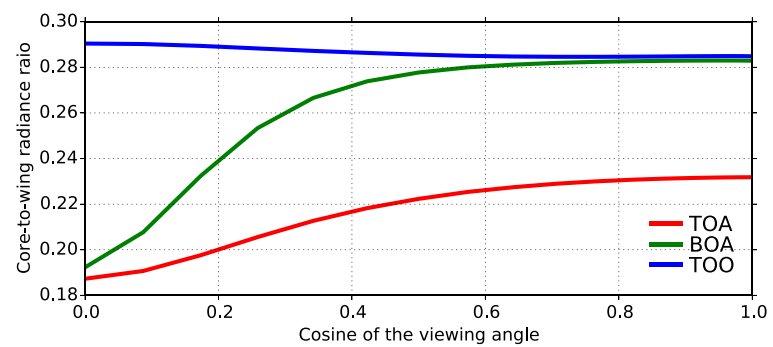

Fig. 5. Core-to-wing radiance ratio for the $H_{\beta}$ Fraunhofer line for the upwelling radiation at the top of atmosphere (TOA), at the bottom of atmosphere (BOA), and just below the ocean surface (TOO) for the overhead sun. (For interpretation of the references to color in this figure caption, the reader is referred to the web version of this paper.)

The broadband spectral structure of fluorescent CDOM components is considered using their so-called reference spectra. The CDOM reference spectra were calculated according to Eq. (40), where $I^{+}(\lambda ; \vartheta ; \varphi)$ and $I^{-}(\lambda ; \vartheta ; \varphi)$ are radiances calculated accounting for the CDOM florescence and neglecting it, respectively. The calculations were performed in the aerosol-free atmosphere accounting for the molecular scattering and gaseous absorption by $\mathrm{O}_{3}$ and $\mathrm{NO}_{2}$ in the whole spectral range for which the CDOM fluorescence properties are available. A high-resolved extraterrestrial solar spectrum reported by Kurucz [42] was used. In particular, the reference spectra were calculated for fulvic and humic acids (according to Hawes [29]) and for seven fluorescent CDOM components identified from natural samples with the PARAFAC analysis (according to Jørgensen et al. [36]). The obtained reference spectra are presented in Fig. 6. One can see that the reference spectra of the various PARAFAC components are clearly distinct from each other. In general, the spectra of the humic-like PARAFAC components have a larger amplitude than the amino acid-like (Fig. 6b and c, respectively). This can be explained by the fact that amino acid-like PARAFAC components exhibit the fluorescence excitation at shorter wavelengths as compared to humiclike, usually below $300 \mathrm{~nm}$ [36]. Hence, even though their fluorescence signal is strong when measured with active techniques, it is much weaker in natural conditions because of a low amount of the electromagnetic radiation reaching the ocean surface in the short-wavelengths region (due to the spectral distribution of the solar radiation and strong ozone absorption in UV). Among the amino acid-like PARAFAC components, fluorescence of component 2 (identified by Jørgensen et al. [36] as tryptophan-like) is noticeably stronger than the others at wavelengths above $320 \mathrm{~nm}$ (Fig. 6c).

Reference spectra of all CDOM components show the noticeable sharp spectral features which demonstrate the filling-in of Fraunhofer lines.

The shapes of humic-like PARAFAC components reference spectra differ significantly from those of humic and fulvic acids calculated using FHA approach (see Fig. 6a). In particular, the fluorescence emission of humic and fulvic acids starts in longer wavelengths and reaches up to $700 \mathrm{~nm}$, which is much longer than for the PARAFAC components. This difference is due to much stronger fluorescence emission functions at longer wavelengths used in the FHA approach. Such strong emission at long wavelengths can be a result of the technique used by Hawes [29] that preferentially extracts material absorbing and fluorescing at longer wavelengths [90]. The reference spectrum of PARAFAC component 1 is abruptly cut at quite high values around $550 \mathrm{~nm}$, which suggests that fluorescence continues further into longer wavelengths, which were not measured by the instrument in the dataset [36] used here.

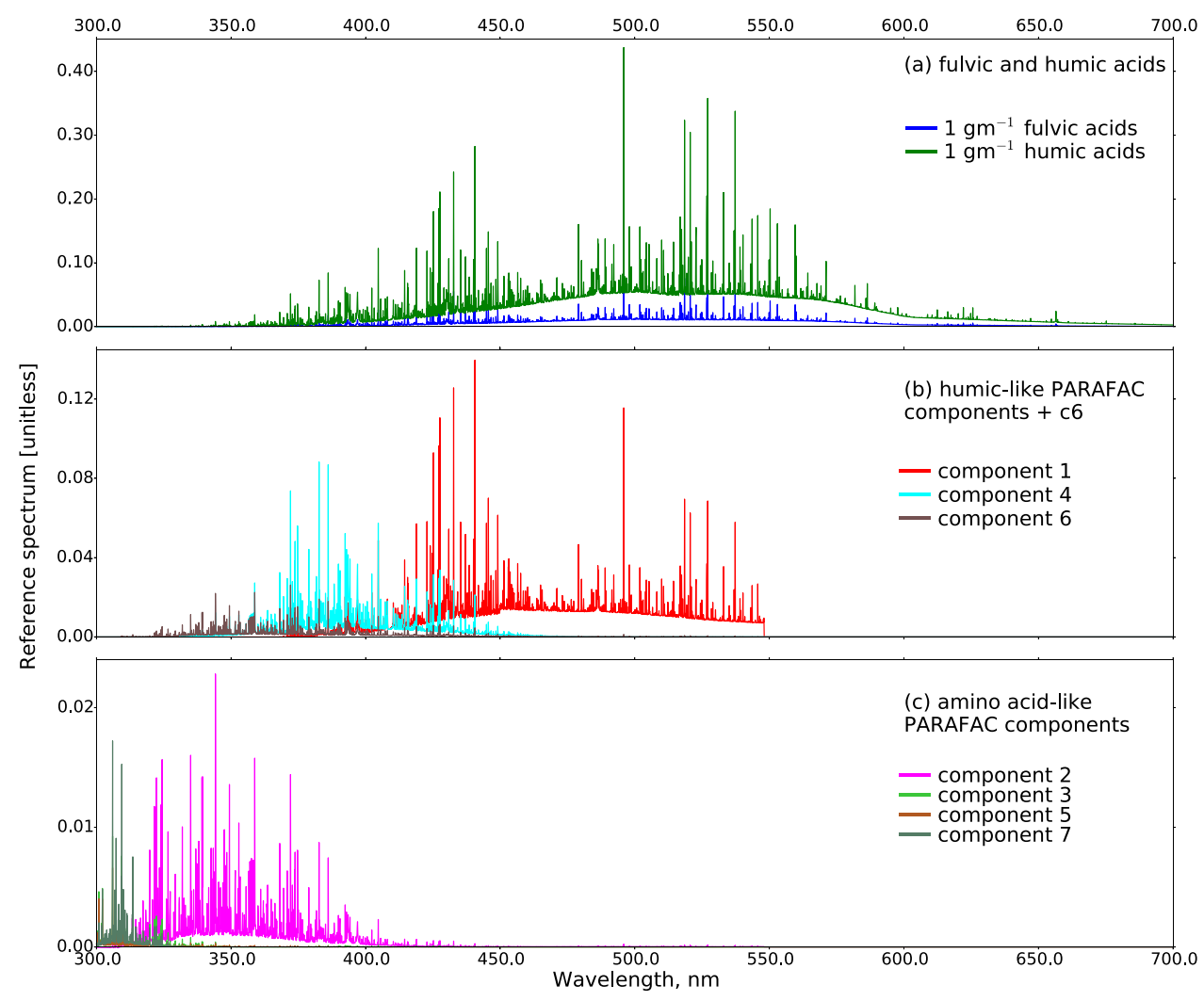

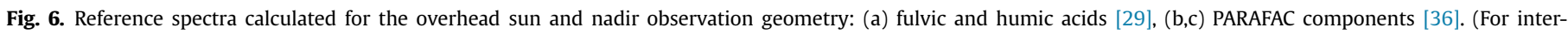
pretation of the references to color in this figure caption, the reader is referred to the web version of this paper.) 


\section{Comparisons to other radiative transfer models}

In this section we present comparisons of SCIATRAN results to those from other radiative transfer models. In particular, we compare the down- and upwelling inelastic fluxes as well as filling-in of $H_{\beta}$ Fraunhofer line in the ocean obtained with Monte Carlo codes [37,47], the radiative transfer model MOMO [64,82], and SCIATRAN.

\subsection{Comparison of inelastic underwater spectral fluxes}

A comparison of results obtained with four ocean radiative transfer models that include VRS by water molecules has been presented by Mobley et al. [47]. The calculations were performed using the Monte Carlo codes with the assumption that the solar beam incident at the sea surface had an irradiance of $1 \mathrm{~W} \mathrm{~m}^{-2} \mathrm{~nm}^{-1}$ at the wavelength of $417 \mathrm{~nm}$, which was selected as the excitation wavelength. There is no atmosphere and no incident solar irradiance at the emission wavelength of $486 \mathrm{~nm}$. Hence, the resulting flux at the emission wavelength is solely due to inelastic scattering at the excitation wavelength. The Rayleigh phase function was used as given in Eq. (A.6). The depolarization ratio $\rho$ was set to 0.17 . The absorption, $a_{w}$, and elastic-scattering, $b_{w}$, coefficients of pure sea water were taken from Smith and Baker [70]:

$a_{w}(417)=0.0156 \mathrm{~m}^{-1}, \quad b_{w}(417)=0.0063 \mathrm{~m}^{-1}$,

$a_{w}(486)=0.0188 \mathrm{~m}^{-1}, \quad b_{w}(486)=0.0032 \mathrm{~m}^{-1}$.

The absorption coefficient, $a_{w}$, was assumed to include the total Raman scattering coefficient. The latter was set to the same value as the elastic scattering coefficient. Thus, the total beam attenuation coefficient at the excitation and emission wavelengths is given by $a_{w}+b_{w}$. The solar zenith angle was set to $60^{\circ}$ and the air-water interface was assumed to be flat. The ocean was considered as an optically semi-infinite medium.

The SCIATRAN calculations presented here use the same scenario but with the ocean depth set to $1000 \mathrm{~m}$ to approximate an optically semi-infinite medium. Under these assumptions the optical thickness of the ocean is about 22. It is sufficient to model the ocean as a semi-infinite medium, because the single scattering albedo, $b_{w} /\left(a_{w}+b_{w}\right)$, is $\sim 0.29$ and $\sim 0.15$ at wavelengths 417 and $486 \mathrm{~nm}$, respectively, and, therefore, the absorption of radiation within the water is strong.

The results obtained with four Monte Carlo codes [47], SCIATRAN, and MOMO model are summarized in Table 1 and Fig. 7. The latter shows the relative differences between SCIATRAN and other RT model simulations given by

Diff $=\frac{F_{\mathrm{SCl}}-F_{\mathrm{RT}}}{F_{\mathrm{SCl}}} \cdot 100$, where $F_{\mathrm{SCI}}$ and $F_{\mathrm{RT}}$ are fluxes calculated using SCIATRAN and other codes, respectively. It follows that SCIATRAN reproduces the irradiances obtained with all Monte Carlo codes very well, especially with Monte Carlo codes denoted by MC2 [37] and MC5 [74,75], for both up- and downwelling fluxes at all considered depths. As can be seen from Fig. 7, the relative differences between SCIATRAN and both MC2 and MC5 results are less than $\sim 0.4 \%$.

The high accuracy of MC2, MC5 and SCIATRAN models is confirmed by additional comparisons of the results with simulations by the independent radiative transfer model MOMO (Table 1, last column) based on the matrix operator method [64,82]. Differences between MOMO and SCIATRAN are at maximum $\sim 0.2 \%$ for downward irradiance at $z=0 \mathrm{~m}$ depth (see right panel of Fig. 7) and even smaller for all other cases.

\subsection{Comparison of Fraunhofer lines filling-in in ocean}

In this section, quantitative comparisons of the modeled fillingin of Fraunhofer lines in the ocean by VRS are presented. As pointed out in Section 4.2 this effect was modeled by Kattawar and $\mathrm{Xu}$ [37] using Monte Carlo simulations, where the filling-in of $H_{\beta}$ and $\mathrm{Mg}$ Fraunhofer lines centered at 486.13 and $518.3 \mathrm{~nm}$, respectively, was investigated. The filling-in effect in SCIATRAN and MOMO models was calculated for $H_{\beta}$ Fraunhofer line according to Eq. (43). To perform comparisons the properties of the atmosphere and ocean were selected in accordance with [37] (see also Section 4.2.1). Note that the large Raman scenario (LRam) as presented in [37] was used for the comparison.

Fig. 8 shows the core-to-wing irradiance ratio for the $H_{\beta}$ Fraunhofer line calculated for the overhead sun using SCIATRAN, MOMO, and Monte Carlo techniques. One can see that SCIATRAN and MOMO simulations are very close to each other with maximum relative differences within $\sim 1 \%$ for both down- and upwelling radiation below the ocean surface. Both models show, however, differences to the Monte Carlo simulations presented in [37] with relative differences of $\sim 3 \%$ and $\sim 2 \%$ for the upward and downward fluxes, respectively. Better agreement between SCIATRAN and MOMO compared to the Monte Carlo simulations can be explained by the fact that the former models used the same solar irradiance (as given in [3]) at the core and wing of $H_{\beta}$ Fraunhofer line whereas the exact values of irradiance used for Monte Carlo simulations were not given by Kattawar and $\mathrm{Xu}$ [37].

\section{Selected comparisons with experimental data}

Comparisons of SCIATRAN modeling results with selected measurements from satellite, ship-based, and underwater instruments are presented in this section. Unless atmospheric and ocean parameters needed for the radiative transfer modeling were

Table 1

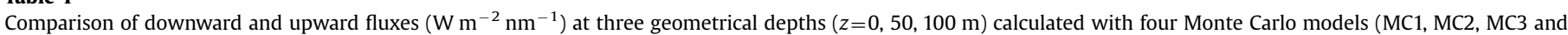
MC5) [47], SCIATRAN, and MOMO [82].

\begin{tabular}{|c|c|c|c|c|c|c|}
\hline$z$ & MC1 & MC2 & MC3 & MC5 & SCIATRAN & MOMO \\
\hline \multicolumn{7}{|c|}{ Downward underwater irradiance } \\
\hline 0 & $1.875 \cdot 10^{-2}$ & $1.874 \cdot 10^{-2}$ & $1.739 \cdot 10^{-2}$ & $1.873 \cdot 10^{-2}$ & $1.873 \cdot 10^{-2}$ & $1.877 \cdot 10^{-2}$ \\
\hline 50 & $2.489 \cdot 10^{-2}$ & $2.488 \cdot 10^{-2}$ & $2.470 \cdot 10^{-2}$ & $2.490 \cdot 10^{-2}$ & $2.493 \cdot 10^{-2}$ & $2.492 \cdot 10^{-2}$ \\
\hline 100 & $1.136 \cdot 10^{-2}$ & $1.136 \cdot 10^{-2}$ & $1.123 \cdot 10^{-2}$ & $1.138 \cdot 10^{-2}$ & $1.140 \cdot 10^{-2}$ & $1.139 \cdot 10^{-2}$ \\
\hline \multicolumn{7}{|c|}{ Upward underwater irradiance } \\
\hline 0 & $3.532 \cdot 10^{-2}$ & $3.512 \cdot 10^{-2}$ & $3.478 \cdot 10^{-2}$ & $3.523 \cdot 10^{-2}$ & $3.518 \cdot 10^{-2}$ & $3.518 \cdot 10^{-2}$ \\
\hline 50 & $1.034 \cdot 10^{-2}$ & $1.042 \cdot 10^{-2}$ & $1.027 \cdot 10^{-2}$ & $1.039 \cdot 10^{-2}$ & $1.039 \cdot 10^{-2}$ & $1.039 \cdot 10^{-2}$ \\
\hline 100 & $0.287 \cdot 10^{-2}$ & $0.296 \cdot 10^{-2}$ & $0.292 \cdot 10^{-2}$ & $0.296 \cdot 10^{-2}$ & $0.297 \cdot 10^{-2}$ & $0.297 \cdot 10^{-2}$ \\
\hline
\end{tabular}



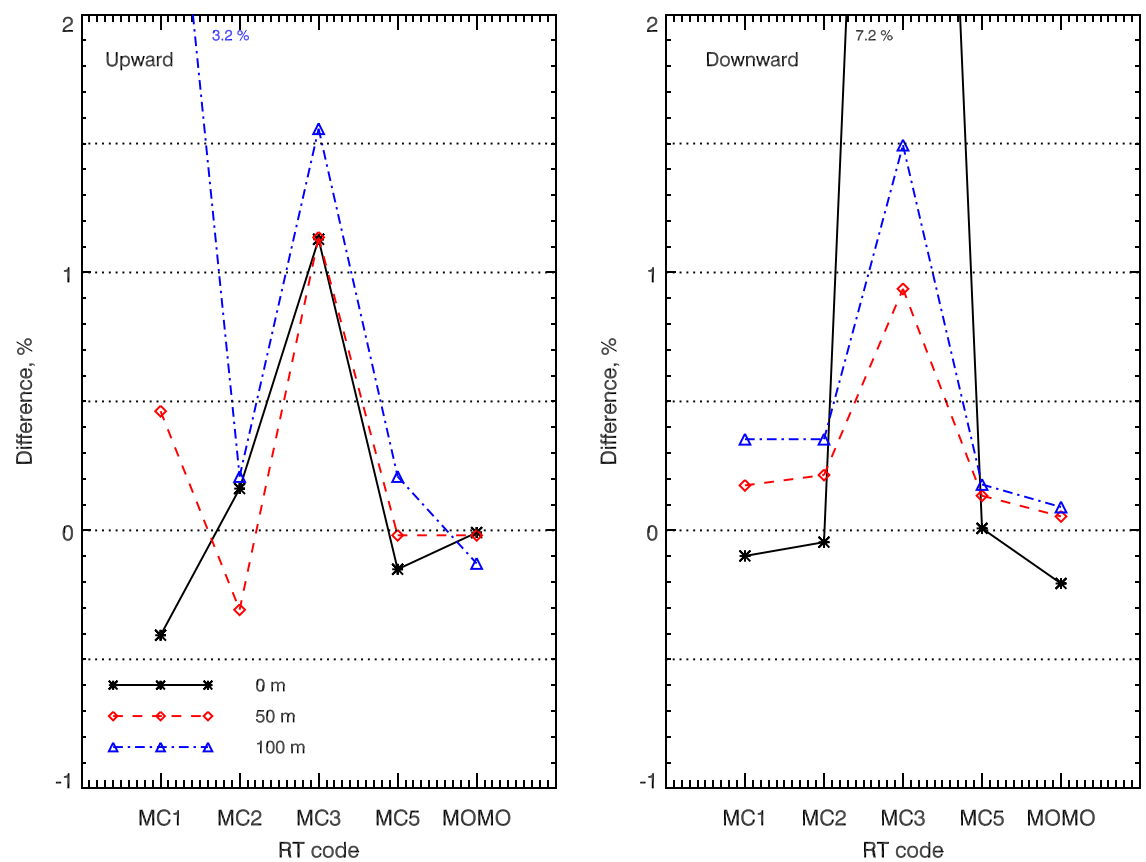

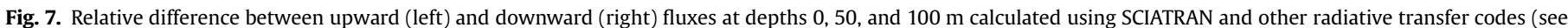
Eq. (47)). (For interpretation of the references to color in this figure caption, the reader is referred to the web version of this paper.)
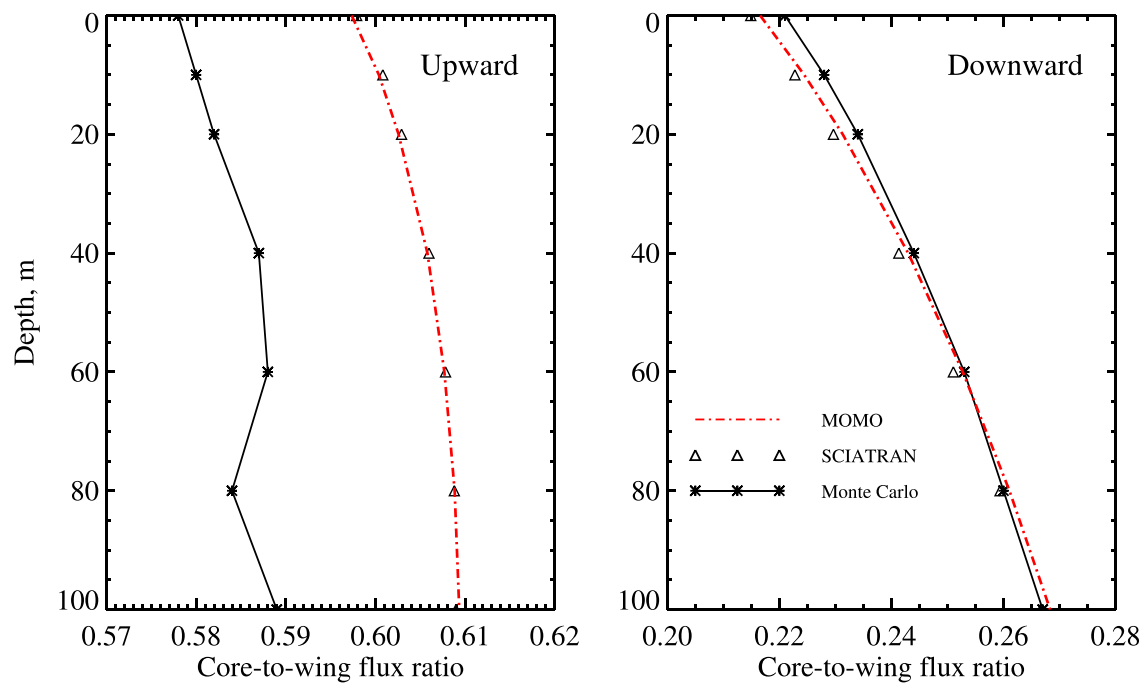

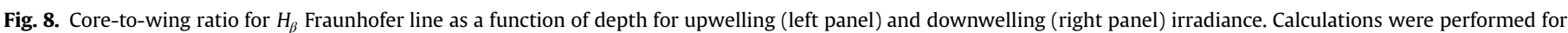
the overhead sun. (For interpretation of the references to color in this figure caption, the reader is referred to the web version of this paper.)

known from the measurements, they were estimated employing a retrieval technique and/or a priori knowledge.

\subsection{Underwater radiation measurements}

The underwater radiation simulated with SCIATRAN was compared to field data collected from a research vessel Polarstern expedition at PS71 Station 281 (15 May 2008 12:00 UTC, 40.81 ${ }^{\circ} \mathrm{N}$, $\left.10.68^{\circ} \mathrm{W}\right)$. The data set comprises measurements of the underwater upwelling and downwelling radiation in the spectral range from 350 to $950 \mathrm{~nm}$ with a spectral resolution of $3.3 \mathrm{~nm}$ and in situ measurements of the temperature, salinity, and chlorophyll concentrations at different depths. Details on the field data acquisition can be found in Bracher et al. [9], the data are publicly available from the PANGAEA database [10].

Simulations were done using the following settings:

Atmosphere: The vertical profiles of temperature, pressure, and concentrations of $\mathrm{O}_{3}$ and $\mathrm{NO}_{2}$ were set according to the Bremen 2D chemical transport model [69] for May at $45^{\circ} \mathrm{N}$. The aerosol optical thickness was set to 0.17 at $550 \mathrm{~nm}$.

Ocean: The underwater profiles of temperature, salinity, and chlorophyll concentration were set in accordance with in situ measurement data.

The total Raman scattering coefficient was set to $2.7 \cdot 10^{-4} \mathrm{~m}^{-1}$ at the reference wavelength of $488 \mathrm{~nm}$. The wavelength dependence of the total Raman scattering coefficient was defined by the resonance contour with the intermediate energy level set to $130 \mathrm{~cm}^{-1}$ (see Appendix A).

The chl- $a$ fluorescence was calculated assuming a Gaussian shape of the emission band with a center at $685 \mathrm{~nm}$ and standard deviation of $10.6 \mathrm{~nm}$. The quantum efficiency was set to $0.8 \%$ (see Appendix B.1).

CDOM fluorescence was calculated using the FHA approach (see Appendix B.2.1). The spectroscopic parameters of the fulvic 


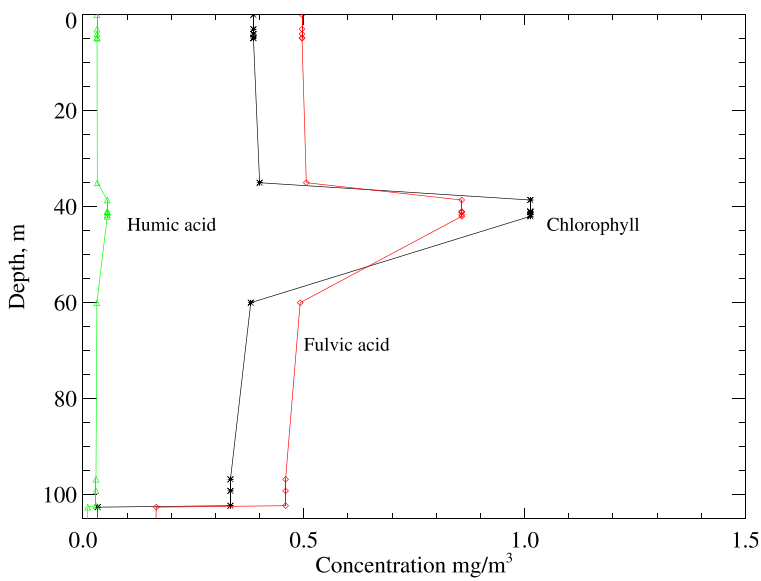

Fig. 9. Concentration profiles of chlorophyll, fulvic acid, and humic acid. (For interpretation of the references to color in this figure caption, the reader is referred to the web version of this paper.)

and humic acids were set according to Table B1. Unfortunately, the measurements of fulvic and humic acid concentrations were not available. Therefore, in order to estimate the concentrations of these acids we calculated the absorption coefficient of CDOM according to the CASE 1 water model of Morel and Maritorena [51]:

$\alpha_{\text {cdom }}(z, \lambda)=0.2\left[\alpha_{\mathrm{w}}\left(\lambda_{0}\right)+0.06 C(z)^{0.65}\right] e^{-S\left(\lambda-\lambda_{0}\right)}$,

where $\alpha_{\mathrm{w}}\left(\lambda_{0}\right)$ is the absorption coefficient of liquid water, $C(z)$ is the chlorophyll concentration at the depth $z$, the spectral slope parameter, $S$, is set to $0.014 \mathrm{~nm}^{-1}$ and $\lambda_{0}=440 \mathrm{~nm}$.

The sum of fulvic and humic acid absorption coefficients is given by

$\alpha_{\mathrm{fha}}(z, \lambda)=C_{\mathrm{f}}(z) \alpha_{\mathrm{f}}(\lambda)+C_{\mathrm{h}}(z) \alpha_{\mathrm{h}}(\lambda)$.

Here, $C_{\mathrm{f}}(z), C_{\mathrm{h}}(z)$, and $a_{\mathrm{f}}(\lambda), a_{\mathrm{h}}(\lambda)$ are concentrations and specific absorption coefficients of fulvic and humic acids given by Eq. (B.12), respectively.

Assuming that fulvic and humic acids are the main components

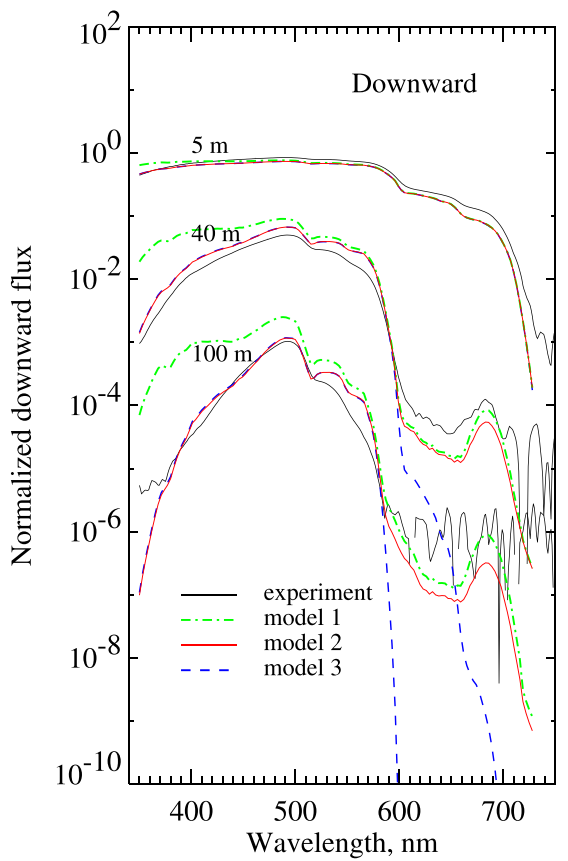

of CDOM, the concentration of acids at a given depth was estimated solving the following minimization problem:

$\left\|\alpha_{\text {cdom }}(z, \lambda)-C_{\mathrm{f}}(z) \alpha_{\mathrm{f}}(\lambda)-C_{\mathrm{h}}(z) \alpha_{\mathrm{h}}(\lambda)\right\|^{2} \longrightarrow \min$,

where $\alpha_{\text {cdom }}(z, \lambda)$ is given by Eq. (48).

The measured concentration of chlorophyll along with estimated concentrations of fulvic and humic acids is presented in Fig. 9.

The spectral down- and upwelling underwater fluxes at three selected depths (5, 40, and $100 \mathrm{~m}$ ) measured in situ and modeled by SCIATRAN are shown in Fig. 10. In order to reduce the dependence of the fluxes on the unknown atmospheric parameters and extraterrestrial solar spectrum, we show in the comparison normalized values, i.e., the measured and simulated underwater fluxes were divided by the corresponding downward fluxes just above the ocean surface.

The underwater spectral fluxes calculated with SCIATRAN using the concentration profiles of fulvic and humic acids, given in Fig. 9, are presented in Fig. 10 by dash-dotted green lines denoted as "model 1". The modeled fluxes overestimate significantly the measured ones especially at the short wavelengths. This demonstrates that the light absorption by acids in the model is too weak and that the CDOM absorption does not follow the CASE I water model assumptions. Assuming the specific absorption coefficients of fulvic and humic acids used for these simulations following Carder et al. [11], we increase absorption by increasing their concentration.

The resulting underwater spectral fluxes calculated with SCIATRAN using 2.5 times larger concentrations of fulvic and humic acid than in "model 1" are presented in Fig. 10 by solid red lines and denoted as "model 2". In contrast to "model 1" the simulated fluxes reproduce the measured fluxes much better at the short wavelengths.

Moreover, the plot reveals that the general behavior of the observed spectral distributions of the underwater irradiance is well reproduced by SCIATRAN for both upwelling and downwelling fluxes. The irradiance is maximal at the wavelength of

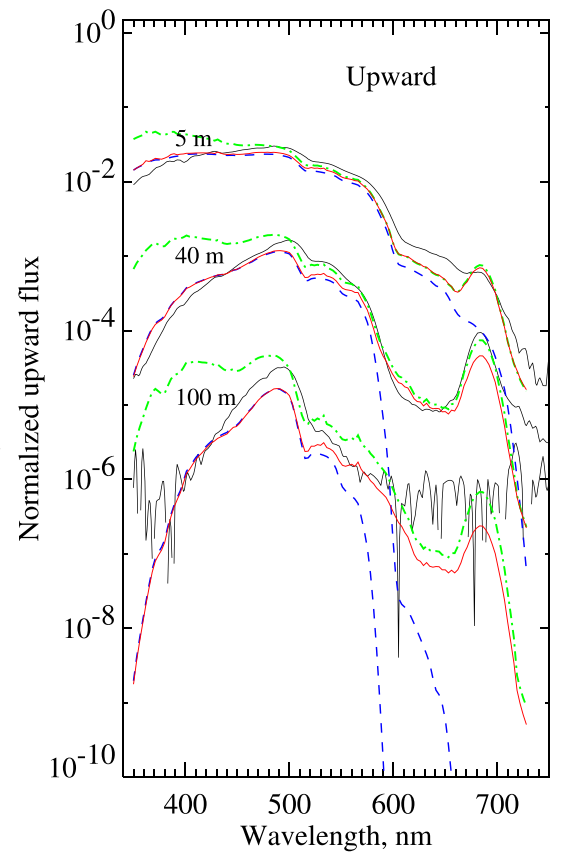

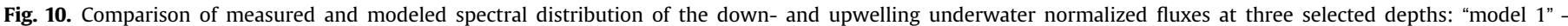

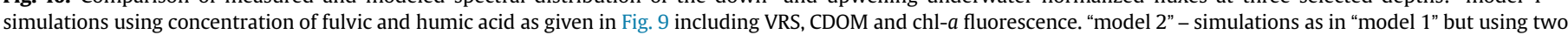

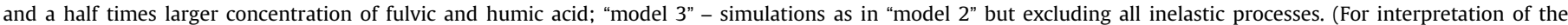
references to color in this figure caption, the reader is referred to the web version of this paper.) 
$\sim 500 \mathrm{~nm}$ and decreases to the shorter and longer wavelengths due to the absorption by chlorophyll, fulvic and humic acids and liquid water. Fluxes calculated neglecting inelastic radiative processes are plotted in Fig. 10 by blue dashed lines and denoted as "model 3 ". It can be seen that nearly all radiation within the ocean at wavelengths larger than $\sim 600 \mathrm{~nm}$ originate from inelastic radiative processes. The local maximum at the wavelength near $685 \mathrm{~nm}$ represents the well known chlorophyll emission band and is well observed at $\sim 40 \mathrm{~m}$ depth where the maximum of chlorophyll concentration is located (see Fig. 9). The irregular oscillations of the measured fluxes, which are clearly seen at the depth of $100 \mathrm{~m}$, are caused by the instrument noise.

The comparisons presented in Fig. 10 clearly demonstrate the ability of the SCIATRAN model to reproduce realistic light field conditions in an open ocean environment with enhanced chlorophyll concentration.

\subsection{Ship-borne measurements of the seawater reflectance}

Numerous measurements of light penetrating clear natural waters were performed during the ship-based TransBrom campaign across the western Pacific in October 2009 [54]. MAX-DOAS (Multi-AXis differential optical absorption spectroscopy) measurements were carried out using a visible spectrometer covering a wavelength range from 400 to $570 \mathrm{~nm}$ with a spectral resolution of $0.8 \mathrm{~nm}$. Among other observation geometries, measurements pointing towards the water surface were taken at elevation angles of $45^{\circ}$ and $60^{\circ}$ with respect to the horizon, and in azimuthal directions of $45^{\circ}$ and $90^{\circ}$ relative to the ship's heading. These measurements are dominated by liquid water effects, and are denoted as water-pointing measurements in the following.

The measured spectra were divided into two groups: clear water measurements and white cap measurements. In the latter case, the observed scene was covered by white caps. A color index was used to characterize the dominant color in the observed scene, and to distinguish between these two groups (see [54] for details). The spectra of the white caps were regarded as the reflection of the incoming light before penetrating the water. Thus, the difference between white caps and undisturbed water measurements was, in first approximation, determined by the spectral effects of liquid water, while any atmospheric impact cancels out. Taking into account that the main radiative processes in the clear water are absorption by liquid water, elastic scattering, and VRS, we can write

$\ln \frac{I(\lambda)}{I_{0}(\lambda)}=-S \sigma(\lambda)-P(\lambda)+v(\lambda)$,

where $I(\lambda)$ and $I_{0}(\lambda)$ are clear water and white cap spectra, respectively, $S$ is the so-called slant column of the liquid water, which is the concentration of absorber integrated along the light path, $\sigma(\lambda)$ is the liquid water cross section [55], $P(\lambda)$ is a fourthorder polynomial accounting for instrumental effects and elastic scattering under water, and $v(\lambda)$ is a residual spectrum containing the VRS effect. The DOAS fit, i.e., the solution of the following minimization problem:

$\left\|\ln \frac{I(\lambda)}{I_{0}(\lambda)}-S \sigma(\lambda)-P(\lambda)\right\|^{2} \longrightarrow \min$,

with respect to the slant column $S$ and polynomial coefficients were performed on all water-pointing measurements throughout the whole campaign. Afterwards, all DOAS residuals, $v(\lambda)$, were averaged and the resulting empirical differential VRS spectrum is plotted in Fig. 11 by the solid black line.

The simulated VRS spectrum was calculated according to the following expression:

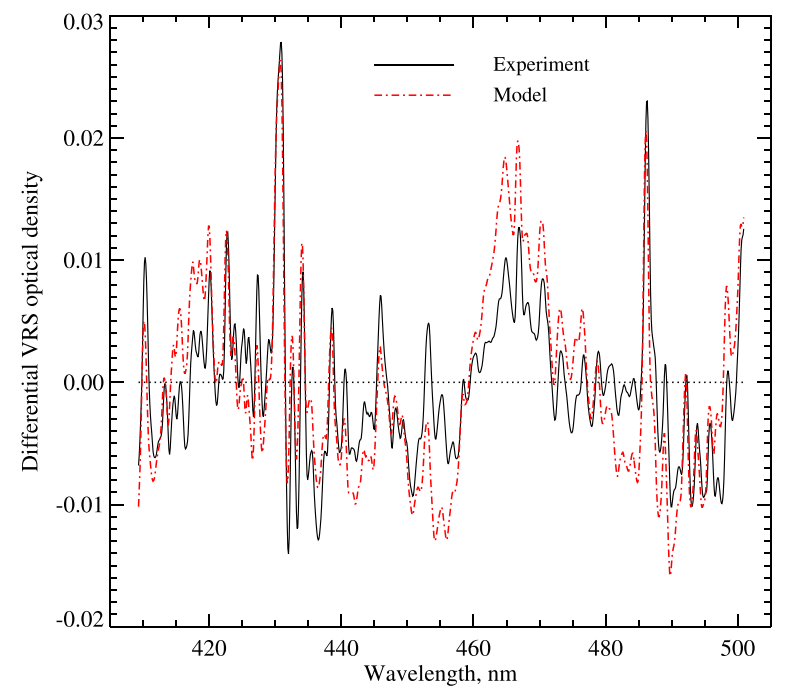

Fig. 11. Experimental and modeled differential VRS spectra. Solid black line - retrieved from MAX-DOAS measurements [54]. Dash-dotted red line - calculated using SCIATRAN with the geometrical settings appropriate to the ground-based measurements performed during the TransBrom campaign. (For interpretation of the references to color in this figure caption, the reader is referred to the web version of this paper.)

$F(\lambda)=\ln \frac{I^{+}(\lambda)}{I^{-}(\lambda)}$,

where $I^{+}(\lambda)$ and $I^{-}(\lambda)$ are modeled water-pointing clear water reflectance spectra calculated including and excluding VRS effect, respectively. The SCIATRAN simulations were performed including all relevant absorption and scattering processes in both atmosphere and ocean. In particular, clear water with low chlorophyll concentration of $0.1 \mathrm{mg} \mathrm{m}^{-3}$ was assumed, which is a realistic scenario for most of the measurement campaign. The resulting spectrum is shown in Fig. 11 by the dash-dotted red line. We note that for the comparison to the empirical spectrum a fourth-order polynomial was subtracted from the simulated VRS spectrum, yielding the simulated differential VRS spectrum.

It follows from Fig. 11 that the differential VRS spectrum retrieved from MAX-DOAS measurements shows very similar features as the simulated one. In particular, one can see that modeled spectrum reproduces the filing-in of numerous Fraunhofer lines and the jump around $\sim 465 \mathrm{~nm}$, whose origin was discussed in Section 4.2.2. Nevertheless, some differences are observed, predominantly in the broadband structures that are larger in the simulated spectrum. The possible reasons of observed differences are discussed by Peters et al. [54] (see Section 4.3 in [54] for details).

\subsection{Hyper-spectral satellite radiation measurements}

In the previous sections we have compared SCIATRAN simulations with the measurements performed in the ocean and just above the ocean surface. Here, we compare modeled and measured VRS spectra at the top of atmosphere using selected hyperspectral measurements from the SCIAMACHY satellite instrument [8,25] over the South Pacific on 23 October 2008 (Orbit: 34764; Pixel: 3152; Lat: $-26.5347^{\circ}$; Lon: $\left.-121.9645^{\circ}\right)$. Considering the difference between the measured and simulated intensities due to unknown atmospheric parameters as a small perturbation and restricting to the linear term of the Taylor series expansion, the following expression for the logarithm of the measured radiance can be written as: 
$\ln I_{\lambda}^{\prime}=\ln I_{\lambda}^{+}+\sum_{i=1}^{N_{a}} \frac{\partial \ln I_{\lambda}^{+}}{\partial p_{i}} \Delta p_{i}+\sum_{i=1}^{N_{o}} \frac{\partial \ln I_{\lambda}^{+}}{\partial q_{i}} \Delta q_{i}+\varepsilon_{\lambda}$,

where $I_{\lambda}^{\prime}$ is the measured intensity, $I_{\lambda}^{+}$is the modeled intensity calculated employing a coupled ocean-atmosphere radiative transfer model including VRS processes, $\lambda$ is the wavelength, $N_{a}$ and $N_{o}$ are the number of atmospheric and oceanic parameters included in the model. $p_{i}$ and $q_{i}$ are the relevant atmospheric and oceanic parameters, and $\Delta p_{i}$ and $\Delta q_{i}$ are their variations, $\varepsilon_{\lambda}$ summarizes the linearization and instrumental errors.

To separate spectral features caused by the VRS effect, the inelastically scattered radiation is presented in the following equivalent form:

$\ln I_{\lambda}^{+}=\ln I_{\lambda}^{-}+V_{\lambda}$,

where $I_{\lambda}^{-}$is the TOA intensity calculated excluding the VRS process, and the additive component $V_{\lambda}$ is referred to the following $[17,83]$ as the VRS reference spectrum:

$V_{\lambda}=\ln \frac{I_{\lambda}^{+}}{I_{\lambda}^{-}}$

Substituting Eq. (55) into the right-hand side of Eq. (54), we get

$\ln I_{\lambda}^{\prime}=\ln I_{\lambda}^{-}+\Delta \ln I_{\lambda}^{-}+V_{\lambda}+\Delta V_{\lambda}+\varepsilon_{\lambda}$

where the operator $\Delta$ denotes the first order perturbation of the intensity logarithm or VRS reference spectrum caused by the perturbations of atmospheric and oceanic parameters $p_{i}$ and $q_{i}$ :

$\Delta=\sum_{i=1}^{N_{o}} \frac{\partial}{\partial q_{i}} \Delta q_{i}+\sum_{i=1}^{N_{a}} \frac{\partial}{\partial p_{i}} \Delta p_{i}$

Eq. (57) constitutes the linear relationship between the measured intensity, on the one hand, and modeled intensity as well as variations of the atmospheric and oceanic parameters, on the other hand. If all parameters can be obtained by solving Eq. (57), the variation of $V_{\lambda}$ can be calculated according to Eq. (58).

Since in real applications it is not possible to retrieve all parameters needed to calculate $\Delta V_{\lambda}$ according to Eq. (58), we need to formulate an adequate approximation. In particular, we assume that:

- Variations of the elastically scattered radiation, $\Delta \ln I_{\lambda}^{-}$, are caused by the variations of all relevant absorbers in the atmosphere and by water and phytoplankton absorption in the ocean.

- All broadband contributions that affect the radiance such as aerosols, optically thin clouds, radiometric calibration errors are compensated for by using a low-order polynomial in a fit routine (see below).

- The variations of the VRS reference spectrum, $\Delta V_{\lambda}$, given by Eq. (58), are caused by variations of oceanic parameters only.

- The total absorption in water can be calculated based on the chlorophyll concentration according to the CASE 1 water model of Morel and Maritorena [51].

A detailed discussion of all introduced approximations is given in Dinter et al. [17]. The following least square minimization problem applied to the wavelength range from 450 to $524 \mathrm{~nm}$ was solved:

$$
\begin{gathered}
\| \ln I_{\lambda}^{\prime}-\ln I_{\lambda}^{-}-V_{\lambda}-q_{V} W_{V}(\lambda)-q_{O c} W_{O c}(\lambda) \\
-q_{R} R(\lambda)-\sum_{i=1}^{M} S_{i} \sigma_{i}(\lambda)-P(\lambda) \|^{2} \rightarrow \min ,
\end{gathered}
$$

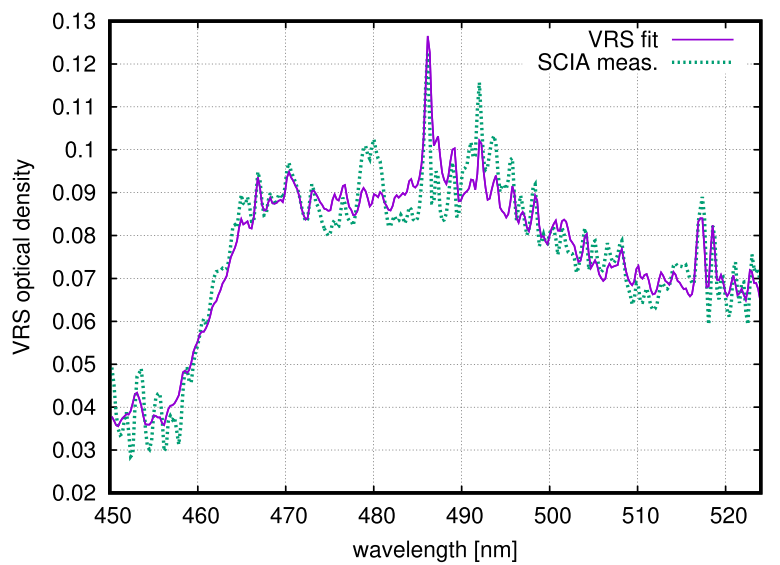

Fig. 12. Comparison of the VRS signal extracted from the SCIAMACHY radiance measurement and the fit of the modeled VRS reference spectrum. (For interpretation of the references to color in this figure caption, the reader is referred to the web version of this paper.)

where $W_{V}(\lambda)$ and $W_{O c}(\lambda)$ are the VRS and oceanic weighting functions introduced in [17], $q_{V}$ and $q_{O c}$ are the appropriate fit factors, $R(\lambda)$ is a rotational Raman scattering reference spectrum (see Eq. (40)), which compensates for the atmospheric Ring effect [26], and $\sigma_{i}(\lambda)$ and $S_{i}$ are the absorption cross sections and slant column densities of considered atmospheric trace gases, specifically water vapor, ozone, glyoxal, oxozone $\left(\mathrm{O}_{4}\right)$ and nitrogen dioxide, $P(\lambda)$ is the third-order polynomial.

The parameters $q_{V}, q_{O c}, q_{R}, S_{i}$, and the polynomial coefficients are the unknown parameters and were obtained by computing the least square minimization solution of Eq. (59). The spectral structures of the VRS signal were extracted from the TOA radiance measurement as follows:

$V_{\lambda}^{\text {mes }}=\ln I_{\lambda}^{\prime}{ }_{\lambda}-\ln I_{\lambda}^{-}-q_{O c} W_{O c}(\lambda)-q_{R} R(\lambda)-\sum_{i=1}^{M} S_{i} \sigma_{i}(\lambda)-P(\lambda)$,

and given in Fig. 12 by the dashed green line. The modeled VRS reference spectrum is calculated then by

$V_{\lambda}^{\bmod }=V_{\lambda}+q_{V} W_{V}(\lambda)$

and given in Fig. 12 by the solid line.

Fig. 12 shows clearly that the retrieved VRS spectral structure was identified in the SCIAMACHY satellite measurements. Similar to the ship-borne measurements (see Section 6.2), the modeled VRS spectrum reproduces the filing-in of Fraunhofer lines and the jump around $\sim 465 \mathrm{~nm}$ as discussed in Section 4.2.2. The fit factor $q_{V}$ of the VRS weighting function contains information about the light availability in ocean and was utilized in Dinter et al. [17] as a proxy for the estimation of amount of light within ocean.

\section{Conclusions}

This paper presents new developments in the radiative transfer model SCIATRAN introduced in [61]. The new version of the model is capable of simulating the main inelastic radiative processes in both atmosphere and ocean. In particular, we have implemented the rotational Raman scattering, vibrational Raman scattering, CDOM and chl-a fluorescence in the coupled ocean-atmosphere radiative transfer solver. Thus, the model is suitable for a wide range of applications related to the remote sensing of the Earth's atmosphere and ocean. The presented study describes the main features of the model as well as basic principles of the radiative transfer problem and of the methodology to solve the radiative 
transfer equation including inelastic radiative processes. Some selected comparisons with other radiative transfer models as well as with satellite, ship-borne, and underwater measured data are provided to illustrate the reliability of the SCIATRAN model.

The study by Wolanin et al. [90] used the presented SCIATRAN package to simulate CDOM fluorescence in ocean waters and confirms the applicability of the model also to case 2 waters. As in the radiative transfer simulations based on matrix-operator by Schröder et al. [64], it was shown that only in case 2 waters with very high CDOM concentrations, CDOM fluorescence can reach or exceed the relevance of VRS at short wavelengths. Schroeder et al. determined that in the water column, depending on optically active substances and on actual depth, VRS can provide 100\% of the light field at wavelengths greater than $580 \mathrm{~nm}$. Across all water types the effect of CDOM fluorescence on depth-dependent irradiances is less significant than the effect of VRS, except for near surface levels and high CDOM concentrations. The different signatures of filling-in of Fraunhofer lines caused by variations in emission spectra among CDOM fluorescing components could be identified in high spectrally resolved satellite data which then could be used to trace the origin (e.g., marine versus terrigenous) of dissolved organic matter. However, for the development of such retrievals precise measurements of these spectra accompanied by correctly determined molar absorbance, quantum efficiency and concentrations of these components and the separation of their filling-in of Fraunhofer lines from VRS and RRS are needed. This SCIATRAN package would be suitable to develop these retrievals as it has been done for the marine chl- $a$ fluorescence in [90].

Generally, the presented software package contains all features of the previous versions of SCIATRAN model and is targeted to users who do not have an extensive knowledge in the field of radiative transfer. The implemented interfaces, default modes, and numerous templates facilitate the usage of a model in applications related to remote sensing of the Earth's atmosphere and ocean.

As before, the further developed SCIATRAN software package along with a detailed User's Guide is freely available for scientists affiliated at public scientific facilities and students via the web page of the Institute of Environmental Physics (IUP), University of Bremen: http://www.iup.physik.uni-bremen.de.

\section{Acknowledgments}

We are grateful to J. von Bismarck (FUB Berlin) for providing the data presented in Table 1 and in Fig. 8 derived using the MOMO code, B. Taylor (AWI) for providing explanations of underwater radiation measurements data presented in Fig. 10, E. Peters (IUP) for the provision and helpful discussions of seawater reflectance measurements presented in Fig. 11, and C. Stedmon (DTV) for providing PARAFAC data.

The development and maintenance of the radiative transfer model SCIATRAN since 2007 was funded in parts by the State of Bremen and the University of Bremen. A. Bracher, T. Dinter and A. Wolanin received funding via the Alfred-Wegener-Institute, AWI Bremerhaven, and ESA SynSenPFT project. This research also supports the objectives of National Grant no. TR 172 (AC)3 "Arctic Amplification" subproject C03.

\section{Appendix A. Vibrational Raman scattering: spectroscopic parameters}

The vibrational Raman scattering coefficient (see Eq. (19)) is implemented into SCIATRAN code as follows:
Table A1

Parameters $\alpha_{i}, \Delta \nu_{i}$ and $\sigma_{i}$ of the Raman frequency redistribution function.

\begin{tabular}{cccc}
\hline$i$ & $\alpha_{i}$ & $\Delta \nu_{i}\left(\mathrm{~cm}^{-1}\right)$ & $\sigma_{i}\left(\mathrm{~cm}^{-1}\right)$ \\
\hline 1 & 0.41 & 3250 & 89.179 \\
2 & 0.39 & 3425 & 74.317 \\
3 & 0.10 & 3530 & 59.453 \\
4 & 0.10 & 3625 & 59.453 \\
\hline
\end{tabular}

$r_{\mathrm{v}}\left(\lambda^{\prime}, \lambda\right)=\beta_{\mathrm{vrs}}\left(\lambda^{\prime}\right) \frac{\nu^{\prime 2}}{k_{\nu}} f^{R}\left(\nu^{\prime}, \nu\right)$,

where $\beta_{\mathrm{vrs}}\left(\lambda^{\prime}\right)$ is the total Raman scattering coefficient expressed in $\left(\mathrm{m}^{-1}\right), \nu^{\prime}=k_{\nu} / \lambda^{\prime}$ and $\nu=k_{\nu} / \lambda$ are excitation and emission wavenumbers $\left(\mathrm{cm}^{-1}\right), \lambda$ and $\lambda^{\prime}$ are wavelengths in nanometer, $k_{\nu}=10^{7} \mathrm{~nm} \mathrm{~cm}^{-1}$, and $f^{R}\left(\nu^{\prime}, \nu\right)$ is the Frequency Redistribution Function (FRF) expressed in (cm).

The FRF of the Raman scattered light is implemented following Haltrin and Kattawar [28] as

$f^{R}\left(\nu^{\prime}, \nu\right)=\frac{1}{N_{R}} \sum_{i=1}^{4} \alpha_{i} \exp \left[-\frac{\left(\nu^{\prime}-\nu-\Delta \nu_{i}\right)^{2}}{2 \sigma_{i}^{2}}\right]$.

$N_{R}=\sqrt{2 \pi} \sum_{i=1}^{4} \alpha_{i} \sigma_{i}$

where parameters $\alpha_{i}, \Delta \nu_{i}$ and $\sigma_{i}$ are given in Table A1, $1 / N_{R}=5.152 \cdot 10^{-3} \mathrm{~cm}$. The FRF is normalized as follows:

$\int_{-\infty}^{\infty} f^{R}\left(\nu^{\prime}, \nu\right) d \nu^{\prime}=\int_{-\infty}^{\infty} f^{R}\left(\nu^{\prime}, \nu\right) d \nu=1$.

We note that the FRF, represented by the four Gaussian components, shows also a temperature dependency. Therefore, parameters given in Table A1 can vary in different publications (see, e.g., $[37,89])$.

The total vibrational Raman scattering coefficient is given as follows:

$\beta_{\mathrm{vrs}}(\lambda)=\frac{1}{4 \pi} \int_{\lambda^{\prime}>\lambda} r\left(\lambda, \lambda^{\prime}\right) \int_{\Omega} \mathcal{P}_{\mathrm{vrs}}\left(\Omega, \Omega^{\prime}\right) d \Omega^{\prime} d \lambda^{\prime}$,

where the integration is performed over all emission wavelengths larger than $\lambda^{\prime}$, and $\mathcal{P}_{\mathrm{vrs}}\left(\Omega, \Omega^{\prime}\right)$ is the vibrational Raman scattering phase function. Following [48], the latter is implemented as

$\mathcal{P}_{\mathrm{vrs}}(\gamma)=\frac{3}{4} \cdot \frac{1+3 \rho}{1+2 \rho}\left[1+\frac{1-\rho}{1+3 \rho} \cos ^{2} \gamma\right]$,

where $\rho$ is the depolarization ratio and $\gamma$ is the scattering angle. We neglect the dependence of $\rho$ on the Raman shift and use in accordance with [22] the average value of $\rho=0.17$ for the Raman scattering in water.

The wavelength dependence of the total vibrational Raman scattering coefficient was investigated among others in [2,5,19]. At wavelengths far from the water absorption band, Raman scattering is expected to follow inverse power law dependence, i.e., $\sim \lambda^{-4}$. Closer to the absorption band, Raman scattering is expected to follow a wavelength dependence of approximately $\sim \nu^{4} /\left(\nu_{i}^{2}-\nu^{\prime 2}\right)^{2}$, where $\nu^{\prime}$ and $\nu$ are frequencies of the incident and scattered photons, respectively, and $\nu_{i}$ is the frequency of the intermediate energy level reached by the incident photon.

Both wavelength dependencies of the total Raman scattering coefficient are implemented in the SCIATRAN code. In particular, one can use one of the following two representations: 
Table A2

Raman cross section to the scattering angle $90^{\circ}$ at $\lambda_{r}=488 \mathrm{~nm}$.

\begin{tabular}{ll}
\hline Cross section $\left(\mathrm{cm}^{2}\right.$ molecule $\left.{ }^{-1} \mathrm{sr}^{-1}\right)$ & Reference \\
\hline$(8.6) \times 10^{-30}$ & Chang and Young [13] \\
$(8.1 \pm 0.7) \times 10^{-30}$ & Romanov and Shuklin [59] \\
$(9.0 \pm 2.0) \times 10^{-30}$ & Kondilenko et al. [40] \\
$(8.2 \pm 1.0) \times 10^{-30}$ & Marshall and Smith [45] \\
$(8.4 \pm 0.5) \times 10^{-30}$ & Bartlett et al. [2] \\
\hline
\end{tabular}

Inverse power law: $\quad \beta_{\mathrm{vrs}}\left(\lambda^{\prime}\right)=\beta_{0}^{R}\left(\frac{\lambda_{r}}{\lambda^{\prime}}\right)^{n}$,

Resonance theory: $\quad \beta_{\mathrm{vrs}}\left(\lambda^{\prime}\right)=\beta_{0}^{R} \frac{1}{N_{r}} \frac{\nu^{4}}{\left(\nu_{i}^{2}-\nu^{\prime 2}\right)^{2}}$,

where $\beta_{0}{ }^{R}$ is the total Raman scattering coefficient at a reference wavelength $\lambda_{r}$, the value of $n$ can be specified by user, $\nu_{i}$ is the relevant intermediate energy level for water equal to $76923.1 \mathrm{~cm}^{-1}$ $\left(\lambda_{i}=130.0 \mathrm{~nm}\right), N_{r}$ is the normalization factor given by

$N_{r}=\frac{\left(\nu_{i}^{2}-\nu_{r}^{\prime 2}\right)^{2}}{\nu_{r}^{4}}, \quad \nu_{r}=\frac{k_{\nu}}{\lambda_{r}}, \quad \nu_{r}^{\prime}=\nu_{r}+\Delta \nu$,

and $\Delta \nu$ is the Raman shift.

The absolute magnitude for $\beta_{\mathrm{vrs}}\left(\lambda_{r}\right)=\beta_{0}^{R}$ was found to take a value of $(2.7 \pm 0.2) \times 10^{-4} \mathrm{~m}^{-1}$ at $\lambda_{r}$ equal to $488 \mathrm{~nm}$. Many authors have also presented the measurement results of the scattering Raman cross section. Corresponding data are presented in Table A2 and can be used as an input parameter in the SCIATRAN code. We remind the reader that following [2,37], the relationship between the total Raman scattering coefficient and Raman cross section is given by

$\beta_{\mathrm{vrs}}(\lambda)=\frac{800 N \pi}{3}\left[\frac{d \sigma(\lambda)}{d \Omega}\right]_{90^{\circ}} \frac{1+2 \rho}{1+\rho}$,

where $N$ is the number of molecules per cubic centimeter and $[d \sigma(\lambda) / d \Omega]_{90^{\circ}}$ is the scattering Raman cross section corresponding to the scattering angle $90^{\circ}$.

Concluding it is worth to notice that the Raman scattering coefficients of seawater and pure water were found to agree within experimental uncertainty, both in their absolute magnitudes and in their spectral characteristics. It is suggested that Raman scattering coefficient of pure water can be used as an adequate approximation for one of seawater to model Raman scattering by seawater in the UV and visible regions.

\section{Appendix B. Fluorescence: spectroscopic parameters}

The differential emission coefficient of the fluorescence (see Eq. (19)) is used in the following form:

$\sigma_{\mathrm{f}}\left(\lambda^{\prime}, \lambda\right)=\alpha_{\mathrm{f}}\left(\lambda^{\prime}\right) C_{\mathrm{f}} f_{\mathrm{f}}\left(\lambda^{\prime}, \lambda\right)$,

where $\alpha_{\mathrm{f}}\left(\lambda^{\prime}\right)$ is a specific absorption coefficient of a fluorescent substance, $C_{\mathrm{f}}$ is a concentration of that substance, $f_{\mathrm{f}}\left(\lambda^{\prime}, \lambda\right)$ is the wavelength redistribution function, $\lambda^{\prime}$ and $\lambda$ are an excitation and emission wavelength, respectively. The wavelength redistribution function is related to the spectral fluorescence quantum efficiency function which is introduced usually as follows [48]:

$\eta_{\mathrm{f}}\left(\lambda^{\prime}, \lambda\right)=\frac{N_{\mathrm{em}}^{\prime}(\lambda)}{N_{\mathrm{abs}}\left(\lambda^{\prime}\right)}$, where $N_{\text {em }}^{\prime}(\lambda)$ and $N_{\text {abs }}\left(\lambda^{\prime}\right)$ are the numbers of photons emitted at $\lambda$ and absorbed at $\lambda^{\prime}$ per unit spectral interval, respectively. The wavelength redistribution function is obtained from $\eta_{\mathrm{f}}\left(\lambda^{\prime}, \lambda\right)$ converting the number of photons in the numerator and denominator in the energy. It follows that

$f_{\mathrm{f}}\left(\lambda^{\prime}, \lambda\right)=\eta_{\mathrm{f}}\left(\lambda^{\prime}, \lambda\right) \frac{\lambda^{\prime}}{\lambda}$.

Integrating the spectral fluorescence quantum efficiency function over all emission wavelengths $\lambda$, we have

$\Phi_{\mathrm{f}}\left(\lambda^{\prime}\right)=\int_{0}^{\infty} \eta_{\mathrm{f}}\left(\lambda^{\prime}, \lambda\right) d \lambda$,

which defines the quantum efficiency (or quantum yield) of fluorescence.

The lifetime for fluorescence processes is in the order of nanoseconds, which is a sufficient time for the emitted photon to lose memory of the direction of the incident photon. This leads to the fact that emission process can be considered with a good accuracy as isotropic. As the fluorescence is not considered as a scattering process, we do not need any fluorescence correction to the scattering coefficient.

In the following subsections we consider expressions for the differential emission coefficients for the fluorescence by chl- $a$ and CDOM implemented in the SCIATRAN model.

\section{B.1. Fluorescence of chl-a}

For the fluorescence by chl- $a$ the quantum efficiency $\Phi_{1}\left(\lambda^{\prime}\right)$ can be considered with a good accuracy as independent of wavelength. Forster and Livingston [21] have found experimentally that like most other fluorescent materials, the quantum efficiency of chl- $a$ is independent of excitation wavelength. Utilizing this fact, it is assumed below that the quantum efficiency of chl- $a$ is a constant in the spectral range $370-690 \mathrm{~nm}$ while outside this band it is set to zero. The value of this constant quantum efficiency ranges from $1 \%$ to $10 \%$ in accordance with Mobley [48]. In particular, the measured quantum efficiency of fluorescence is a function of the organism and of the photoadaptive state and ranges between $0.2 \%$ and $2.0 \%$ for the cultures studied by Collins et al. [15]. To describe the wavelength redistribution function of the fluorescence by chl- $a$ we use the following approximation [28]:

$f_{1}\left(\lambda^{\prime}, \lambda\right)=\eta_{1}\left(\lambda^{\prime}, \lambda\right) \frac{\lambda^{\prime}}{\lambda} \approx \Phi_{1} f_{1}^{\mathrm{ex}}\left(\lambda^{\prime}\right) f_{1}^{\mathrm{em}}(\lambda) \frac{\lambda^{\prime}}{\lambda}$,

where $\Phi_{1}$ is a constant quantum efficiency of fluorescence, $f_{1}^{\text {ex }}\left(\lambda^{\prime}\right)$ and $f_{1}^{\text {em }}(\lambda)$ are excitation and emission functions, respectively. These functions are implemented in the following form:

$f_{1}^{\mathrm{em}}(\lambda)=\frac{1}{\sqrt{2 \pi} \sigma_{1}} \exp \left[-\frac{\left(\lambda-\lambda_{1}^{\circ}\right)^{2}}{2 \sigma_{1}^{2}}\right]$,

$f_{1}^{\text {ex }}(\lambda)= \begin{cases}1, & 370 \leq \lambda \leq 690 \\ 0, & \text { elsewhere }\end{cases}$

$\lambda^{\circ}{ }_{1}=685 \mathrm{~nm}, \quad \sigma_{1}=10.6 \mathrm{~nm}$,

where $10.6 \mathrm{~nm}$ standard deviation of the Gaussian function corresponds to the value of $25 \mathrm{~nm}$ for the full with at half maximum of the emission band.

Substituting all obtained results into Eq. (B.1), we have

$\sigma_{1}\left(\lambda^{\prime}, \lambda\right)=\Phi_{1} \alpha_{1}\left(\lambda^{\prime}\right) C_{1} f_{1}^{\mathrm{ex}}\left(\lambda^{\prime}\right) f_{1}^{\mathrm{em}}(\lambda) \frac{\lambda^{\prime}}{\lambda}$, 
where $\alpha_{1}\left(\lambda^{\prime}\right)$ and $C_{1}$ are specific absorption coefficient and chlorophyll concentration expressed in $\left(\mathrm{m}^{2} \mathrm{mg}^{-1}\right)$ and $\left(\mathrm{mg} \mathrm{m}^{-3}\right)$, respectively.

We note that the expression for $\sigma_{1}\left(\lambda^{\prime}, \lambda\right)$ given by Eq. (B.9) is the same as reported by Mobley [48] and very similar to that given by Gordon [24] and Haltrin and Kattawar [28] which contains in the denominator of Eq. (B.9) the constant value $\lambda^{\circ}{ }_{1}$ instead of $\lambda$. This difference, however, is not significant because the fluorescence emission band of chl- $a$ is very narrow as compared to the broad excitation wavelength range.

\section{B.2. Fluorescence of $C D O M$}

Two approaches to model CDOM fluorescence, which account for more than one CDOM component (fulvic and humic acids approach, FHA, and additive fluorescent components approach, AFC), are implemented in SCIATRAN.

\section{B.2.1. Fulvic and humic acids approach}

A quantitative approximation for the fluorescence efficiency function $\left(\eta\left(\lambda^{\prime}, \lambda\right)\right)$ of fulvic and humic acids has been suggested by Hawes et al. [30]. This function is defined as the number of quanta fluoresced per $\mathrm{nm}$ interval of emission wavelength divided by the number of quanta absorbed at the excitation wavelength. The expression obtained by fitting the experimental data has the following form:

$\eta\left(\lambda^{\prime}, \lambda\right)=A_{0}\left(\lambda^{\prime}\right) \exp \left[-\left(\frac{1 / \lambda-A_{1} / \lambda^{\prime}-B_{1}}{0.6\left(A_{2} / \lambda^{\prime}+B_{2}\right)}\right)^{2}\right]$,

where model parameters $A_{0}\left(\lambda^{\prime}\right), A_{1}, A_{2}, B_{1}$, and $B_{2}$ are given in a tabular form, $\lambda^{\prime}$ and $\lambda$ are excitation and emission wavelengths, respectively.

Assuming that CDOM consists of fulvic and humic acids only, we obtain the CDOM efficiency function, $\eta_{\mathrm{g}}\left(\lambda^{\prime}, \lambda\right)$, as follows:

$\eta_{\mathrm{g}}\left(\lambda^{\prime}, \lambda\right)=\frac{C_{\mathrm{f}} a_{\mathrm{f}}\left(\lambda^{\prime}\right) \eta_{\mathrm{f}}\left(\lambda^{\prime}, \lambda\right)+C_{\mathrm{h}} a_{\mathrm{h}}\left(\lambda^{\prime}\right) \eta_{\mathrm{h}}\left(\lambda^{\prime}, \lambda\right)}{C_{\mathrm{f}} a_{\mathrm{f}}\left(\lambda^{\prime}\right)+C_{\mathrm{h}} a_{\mathrm{h}}\left(\lambda^{\prime}\right)}$,

where subscripts $f, h$, and $g$ denote fulvic acid, humic acid, and CDOM, respectively, $C_{\mathrm{f}}$ and $C_{\mathrm{h}}$ are concentrations of acids $\left(\mathrm{g} \mathrm{m}^{-3}\right)$, $a_{\mathrm{f}}\left(\lambda^{\prime}\right)$ and $a_{\mathrm{h}}\left(\lambda^{\prime}\right)$ are specific absorption coefficients $\left(\mathrm{m}^{2} \mathrm{~g}^{-1}\right)$. The approximation of specific absorption coefficient has been suggested by Carder et al. [11] in the following form:

$a_{x}\left(\lambda^{\prime}\right)=a_{x}\left(\lambda_{r}\right) \exp \left[S_{x}\left(\lambda_{r}-\lambda\right)\right]$,

where $\lambda_{r}=450 \mathrm{~nm}$ is the reference wavelength, $a_{x}\left(\lambda_{r}\right)$ is the specific absorption coefficient at this wavelength, and $S_{x}$ is the spectral slope parameter $\left(\mathrm{nm}^{-1}\right)$. The estimated values of these parameters obtained from measurement at two stations are given in Table B1. Examples of measured concentrations of fulvic and humic acids are given in Table B2.

The wavelength redistribution function of CDOM fluorescence is given then by

$f_{2}\left(\lambda^{\prime}, \lambda\right)=\eta_{\mathrm{g}}\left(\lambda^{\prime}, \lambda\right) \frac{\lambda^{\prime}}{\lambda}$,

Table B1

Parameters $a_{x}\left(\lambda_{r}\right)$ and $\mathrm{S}_{x}$ for fulvic acid from two stations and humic acid from one station in the Gulf of Mexico according to [11].

\begin{tabular}{llcc}
\hline Station & Type & $a_{x}\left(\lambda_{r}\right)\left(\mathrm{m}^{2} \mathrm{~g}^{-1}\right)$ & $S_{x}\left(\mathrm{~nm}^{-1}\right)$ \\
\hline Mississippi plume & Fulvic & $0.007 \pm 0.001$ & 0.0194 \\
Gulf loop intrusion & Fulvic & $0.005 \pm 0.001$ & 0.0184 \\
& Humic & $0.1302 \pm 0.00005$ & 0.0110 \\
\hline
\end{tabular}

Table B2

Concentrations of fulvic and humic acids from different stations according to [11].

\begin{tabular}{lcccc}
\hline Station & Depth & Chl- $a$ & Humic $\left(\mathrm{g} \mathrm{m}^{-3}\right)$ & Fulvic \\
\hline Mississippi plume & 3 & 1.35 & 0.114 & 0.550 \\
& 3 & 1.20 & 0.126 & 1.270 \\
& 4 & 7.90 & 0.176 & 0.904 \\
& & & & \\
Gulf loop intrusion & 20 & 0.24 & 0.026 & 0.724 \\
& 20 & 0.21 & 0.003 & 0.190 \\
& 20 & 0.08 & 0.014 & 0.234 \\
& 55 & 0.08 & 0.009 & 0.058 \\
Yucatan & & & & \\
Campeche & 10 & 0.06 & 0.029 & 0.223 \\
& 5 & 0.20 & 0.105 & 0.754 \\
Cape San & & & & \\
Blas & 4 & 0.40 & 0.336 & 0.577 \\
& 55 & 1.06 & 0.010 & 0.139 \\
\hline
\end{tabular}

and the differential emission coefficient for CDOM fluorescence is obtained as

$\sigma_{2}\left(\lambda^{\prime}, \lambda\right)=a_{\mathrm{g}}\left(\lambda^{\prime}\right) \eta_{\mathrm{g}}\left(\lambda^{\prime}, \lambda\right) \frac{\lambda^{\prime}}{\lambda}$,

$a_{\mathrm{g}}\left(\lambda^{\prime}\right)=C_{\mathrm{f}} a_{\mathrm{f}}\left(\lambda^{\prime}\right)+C_{\mathrm{h}} a_{\mathrm{h}}\left(\lambda^{\prime}\right)$.

The fluorescence part of the CDOM absorption coefficient is given by

$\alpha_{2}\left(\lambda^{\prime}\right)=a_{\mathrm{g}}\left(\lambda^{\prime}\right) \lambda^{\prime} \int_{\lambda>\lambda^{\prime}} \eta_{\mathrm{g}}\left(\lambda^{\prime}, \lambda\right) \frac{d \lambda}{\lambda}$.

\section{B.2.2. Additive fluorescent components approach}

In addition to the FHA approach described above, a novel approach to model CDOM fluorescence has been implemented into the SCIATRAN code, which is based on PARAllel FACtor (PARAFAC) analysis. PARAFAC is widely applied in the interpretation of excitation-emission matrix fluorescence spectroscopy (EEMS) measurements of CDOM fluorescence to separate the mixture of measurements into the contributions from the underlying individual fluorescent components (see [76] for details).

The PARAFAC model is conceptually similar to the principal component analysis, but decomposes the data matrix $\mathbf{x}$ into a set of trilinear terms (matrices $\mathbf{b}, \mathbf{c}, \mathbf{d}$ ) and a residual array $\varepsilon_{i j k}$ as follows:

$x_{i j k}=\sum_{n=1}^{N} b_{i n} c_{j n} d_{k n}+\varepsilon_{i j k}$,

$i=1, \ldots, I ; \quad j=1, \ldots, J ; \quad k=1, \ldots, K$,

where $x_{i j k}$ is the intensity of fluorescence for the $i$ th sample at emission wavelength $j$ and excitation wavelength $k, b_{\text {in }}$ is directly proportional to the concentration of the $n$th component in sample $i, c_{j n}$ is linearly related to the fluorescence quantum efficiency of the $n$th component at emission wavelength $j, d_{k n}$ is linearly proportional to the specific absorption coefficient at excitation wavelength $k . I, J, K$, and $N$ denote the number of samples, emission wavelengths, excitation wavelengths, and the number of components in the model, respectively. A residual $\varepsilon_{i j k}$ represents the variability, which is not accounted for by the model. The parameters of the PARAFAC model are found by the least-square minimization. 
Matrices $\mathbf{b}, \mathbf{c}$ and $\mathbf{d}$ are used to represent CDOM concentration, fluorescence emission function and specific absorption coefficient, respectively. Since only absorption that causes fluorescence emission is measured in the EEMS, the fluorescence excitation function can be assumed to be constant in the spectral range of the excitation wavelengths.

The PARAFAC model terms are only proportional to the molar absorbance, quantum efficiency and concentration of the fluorescent CDOM components, and hence the matrices need to be scaled appropriately to account for this proportionality. Although in the current version of the SCIATRAN code seven components from [36] were implemented, in general any PARAFAC matrix can be applied as input in the model, together with the corresponding scaling factors.

\section{References}

[1] Barichello LB, Garcia RDM, Siewert CE. Particular solutions for the discrete-ordinates method. J Quant Spectrosc Radiat Transf 2000;64:219-226.

[2] Bartlett JS, Voss KJ, Sathyendranath S, Vodacek A. Raman scattering by pure water and seawater. Appl Opt 1998;37(15):3324-3332.

[3] Beckers JM, Bridges CA, Gilliam LB. A high resolution spectral atlas of the solar irradiance from 380 to 700 nanometers. Technical report, AFGL-TR-76-0126 (U. S. Air Force Geophysics Laboratory), Hanscom Air Force Base, MA, 1976.

[4] Behrenfeld MJ, Westberry TK, Boss ES, O'Malley RT, Siegel DA, Wiggert JD, et al. Satellite-detected fluorescence reveals global physiology of ocean phytoplankton. Biogeosciences 2009;6(5):779-794.

[5] Bischel WK, Black G. Wavelength dependence of Raman scattering cross sections from 200-600 nm. In: Rhodes CK, Egger H, Pummer H, editors. Excimer lasers-1983. New York: American Institute of Physics Edition; 1983. p. 181-187.

[6] Blum M, Rozanov VV, Burrows JP, Bracher A. Coupled ocean-atmosphere radiative transfer model in framework of software package SCIATRAN: selected comparisons to model and satellite data. Adv Space Res 2012;49:1728-1742.

[7] Born M, Wolf E. Principles of optics. 2nd ed. Oxford, London, Edinburgh, New York, Paris, Frankfurt: Pergamon Press; 1964.

[8] Bovensmann H, Burrows JP, Buchwitz M, Frerick J, Noël S, Rozanov VV, et al. SCIAMACHY: mission objectives and measurement modes. J Atmos Sci 1999;56 (2):127-149.

[9] Bracher A, Taylor MH, Taylor B, Dinter T, Röttgers R, Steinmetz F. Using empirical orthogonal functions derived from remote-sensing reflectance for the prediction of phytoplankton pigment concentrations. Ocean Sci 2015;11:139-158.

[10] Bracher A, Taylor MH, Taylor BB, Dinter T, Röttgers R, Steinmetz F. Downwelling spectral irradiance as measured in different depths, remote sensing reflectance and phytoplankton pigment concentration during POLARSTERN cruises ANT-XXIV/1, ANT-XXIV/4, ANT-XXVI/4 and Maria S. Merian cruise MSM18/3 2015.

[11] Carder KL, Steward RG, Harvey GR, Ortner PB. Marine humic and fulvic acids: their effects on remote sensing of ocean chlorophyll. Limnol Oceanogr 1989;34 (1):68-81.

[12] Chandrasekhar S. Radiative transfer.London: Oxford University Press; 1950.

[13] Chang CH, Young LA. Seawater temperature measurement from Raman spectra. Research note 920, n62269-72-c-0204. Washington, DC: Advanced Research Projects Agency; July 1972.

[14] Coble PG. Marine optical biogeochemistry: the chemistry of ocean color. ChemInform 2007;38(20).

[15] Collins DJ, Kiefer DA, SooHoo JB, McDermid IS. The role of reabsorption in the spectral distribution of phytoplankton fluorescence emission. Deep-Sea Res 1985:32:983-1003.

[16] de Beek R, Vountas M, Rozanov VV, Richter A, Burrows J. The Ring effect in the cloudy atmospheres. Geophys Res Lett 2001;28(4):721-724.

[17] Dinter T, Rozanov VV, Burrows JP, Bracher A. Retrieving the availability of light in the ocean utilising spectral signatures of vibrational Raman scattering in hyper-spectral satellite measurements. Ocean Sci 2015;11(3):373-389.

[18] G Falkowski P, Kolber Z. Variations in chlorophyll fluorescence yields in phytoplankton in the world oceans. Funct Plant Biol 1995;22(2):341-355.

[19] Faris GW, Copeland RA. Wavelength dependence of the Raman cross section for liquid water. Appl Opt 1997;36(12):2686-2688.

[20] Fell F, Fischer J. Numerical simulation of the light field in the atmosphereocean system using the matrix-operator method. J Quant Spectrosc Radiat Transf 2001;69:351-388.

[21] Forster LS, Livingston R. The absolute quantum yields of the fluorescence of chlorophyll solutions. J Chem Phys 1952;20:1315-1320.

[22] Ge Y, Gordon HR, Voss KJ. Simulation of inelastic-scattering contributions to the irradiance field in the ocean: variation in Fraunhofer line depths. Appl Opt 1993;32(21):4028-4036.

[23] Gordon HR. Simple calculation of the diffuse reflectance of ocean. Appl Opt $1973 ; 12: 2803-2804$.

[24] Gordon HR. Diffuse reflectance of the ocean: the theory of its augmentation by chlorophyll a fluorescence at $685 \mathrm{~nm}$. Appl Opt 1979;18:1161-1166.
[25] Gottwald M, editor. SCIAMACHY, Monitoring the Changing Earth's Atmosphere. DLR, Institute für Methodik der Fernerkundung; 2006.

[26] Grainger JF, Ring J. Anomalous Fraunhofer line profiles. Nature 1962;93 (4817):762.

[27] Grossmann K, Frieß U, Peters E, Wittrock F, Lampel J, Yilmaz S, et al. Iodine monoxide in the western Pacific marine boundary layer. Atmos Chem Phys 2013; 13:3363-3378.

[28] Haltrin VI, Kattawar GW. Self-consistent solutions to the equation of transfer with elastic and inelastic scattering in oceanic optic. Appl Opt 1993;32 (27):5356-5367.

[29] Hawes SK. Quantum fluorescence efficiencies of marine fulvic and humic acids [M.Sc. thesis]. University of South Florida; 1992.

[30] Hawes SK, Carder KL, Harvey GR. Quantum fluorescence efficiencies of fulvic and humic acids: effects on ocean color and fluorometric detection. In: SPIE ocean optics vol. 1750 of XI, 1992. p. 212-23.

[31] He X, Bai Y, Zhu Q, Gong F. A vector radiative transfer model of coupled oceanatmosphere system using matrix-operator method for rough sea-surface. J Quant Spectrosc Radiat Transf 2010;111:1426-1448.

[32] Jin Z, Charlock TP, Rutledge K, Stamnes K, Wang Y. Analytical solution of radiative transfer in the coupled atmosphere-ocean system with a rough surface. Appl Opt 2006;45(28):7443-7455.

[33] Joiner J, Bhartia PK. The determination of cloud pressure from rotational Raman scattering in satellite backscatter ultraviolet measurements. J Geophys Res 1995;100:23,019-23,026.

[34] Joiner J, Bhartia PK, Cebula RP, Hilsenrath E, McPeters RD, Park H. Rotational Raman scattering (Ring effect) in satellite backscatter ultraviolet measurements. Appl Opt 1995;21:4513-4525.

[35] Joiner J, Vasilkov AP, Flittner DE, Gleason JF, Bhartia PK. Retrieval of cloud pressure and oceanic chlorophyll content using Raman scattering in gome ultraviolet spectra. J Geophys Res: Atmos 2004;109(D1) [D01109].

[36] Jørgensen L, Colin SA, Theis K, Markager S, Middelboe M, Søndergaard M. Global trends in the fluorescence characteristics and distribution of marine dissolved organic matter. Mar Chem 2011;126(1-4):139-148.

[37] Kattawar GW, Xu X. Filling in of Fraunhofer lines in the ocean by Raman scattering. Appl Opt 1992;31(30):6491-6500.

[38] Kattawar GW, Young AT, Humphreys TJ. Inelastic scattering in planetary at mospheres I the Ring effect, without aerosols. Astrophys J 1981;243:10491057.

[39] Kneizys FX, Shettle EP, Abreu LW, Chetwynd JH, Anderson GP, Gallery WO, et al. Users Guide to LOWTRAN 7. Technical Report. Environmental research papers, No. 1010, Air Force Geophysics Laboratory; 1986.

[40] Kondilenko II, Korotkov PA, Klimenko VA, Demyanenko OP. Transverse cross section of Raman scattering of the $\nu_{1}$ vibration of the water molecule in the liquid and gaseous state. Opt Spectrosc (USSR) 1977;43:384-386.

[41] Korn GA, Korn TM. Mathematical handbook for scientists and engineers.New York, San Francisco, Toronto, London, Sydney: McGraw-Hill Book Company; 1968.

[42] Kurucz HL. The solar spectrum: atlases and line identifications. In: Sauval AJ Blomme R, Grevesse N, editors. Laboratory and astronomical high resolution spectra, ASP Conference Series, vol. 81; 1995. p. 17-31.

[43] Landgraf J, Hasekamp OP, van Deelen R, Aben I. Rotational Raman scattering of polarized light in the Earth atmosphere: a vector radiative transfer model using the radiative transfer perturbation theory approach. J Quant Spectrosc Radiat Transf 2004;87:399-433.

[44] Long DA. Raman spectroscopy.London, UK: McGraw-Hill Inc.; 1977.

[45] Marshall BR, Smith RC. Raman scattering and in-water ocean optical properties. Appl Opt 1990;29(1):71-84.

[46] Mayer B, Kylling A. Technical note: the libRadtran software package for radiative transfer calculations - description and examples of use. Atmos Chem Phys 2005:5:1855-1877.

[47] Mobley C, Gentili B, Gordon H, Jin Z, Kattawar G, Morel A, et al. Comparison of numerical models for computing underwater light fields. Appl Opt 1993;32 (36):7484-7504.

[48] Mobley CD. Light and water. Radiative transfer in natural waters. Academic Press, Inc.; 1994.

[49] Mobley CD, Sundman LK. Hydrolight-ecolight 5.0 users' guide. Technical report. Bellevue, WA: Sequoia Scientific, Inc.; 2008.

[50] Mobley CD. Polarized reflectance and transmittance properties of windblown sea surfaces. Appl Opt 2015;54(May (15)):4828-4849.

[51] Morel A, Maritorena S. Bio-optical properties of oceanic waters: a reappraisal. J Geophys Res 2001;106(C4):7163-7180.

[52] Nakajima T, Tanaka M. Effect of wind generated waves on the transfer of solar radiation in the atmosphere-ocean system. J Quant Spectrosc Radiat Transf 1983;29:521-537.

[53] Noxon J, Goody R. Noncoherent scattering of skylight. Atmos Ocean Phys $1965 ; 1: 163-166$.

[54] Peters E, Wittrock F, Richter A, Alvarado LMA, Rozanov VV, Burrows JP. Liquid water absorption and scattering effects in DOAS retrievals over oceans. Atmos Meas Techn 2014; 7:4203-4221.

[55] Pope RM, Fry ES. Absorption spectrum 380-700 nm of pure water: II. Integrating cavity measurements. Appl Opt 1997;36:8710-8723.

[56] Qin Y, Box MA, Jupp DLB. Particular solution of the discrete-ordinate method. Appl Opt 2004;43:3717-3725.

[57] Richter A, Begoin M, Hilboll A, Burrows JP. An improved $\mathrm{NO}_{2}$ retrieval for the GOME-2 satellite instrument. Atmos Meas Techn 2011;4:1147-1159. 
[58] Richter A, Wagner T. The use of UV Visible and near IR solar back scattered radiation to determine trace gases. Physics of earth and space environments. Springer-Verlag; 2011. p. 67-121 [Chapter 2].

[59] Romanov NP, Shuklin VS. Raman scattering cross section of liquid water. Opt Spectrosc (USSR) 1975;38:646-648.

[60] Rozanov VV, Diebel D, Spurr RJD, Burrows JP. GOMETRAN: a radiative transfer model for the satellite project GOME, the plane-parallel version. J Geophys Res 1997; 102(D14):16,683-16,695.

[61] Rozanov VV, Rozanov AV, Kokhanovsky AA, Burrows JP. Radiative transfer through terrestrial atmosphere and ocean: software package SCIATRAN. J Quant Spectrosc Radiat Transf 2014;133:13-71.

[62] Rozanov VV, Vountas M. Radiative transfer equation accounting for rotationa Raman scattering and its solution by the discrete-ordinates method. J Quant Spectrosc Radiat Transf 2014;133:603-618.

[63] Sathyendranath S, Platt T. Ocean-color model incorporating transspectral processes. Appl Opt 1998:37(12):2216-2227.

[64] Schröder M, Barth H, Reuter R. Effect of inelastic scattering on underwater daylight in the ocean: model evaluation, validation, and first results. Appl Opt 2003;42(21):4244-4260.

[65] Schönhardt A, Richter A, Wittrock F, Kirk H, Oetjen H, Roscoe HK, et al. Observations of iodine monoxide columns from satellite. Atmos Chem Phys 2008;8:637-653.

[66] Shefov NN. Intensities of some twilight and night airglow emissions. In: Krassovsky, editor. Spectroscopic, electrophotometrical, and radar researches of aurorae and airglow, vol. IV. Moscow: USSR Acad Sci Publ House; 1959. p. 25-29.

[67] Siewert CE. A concise and accurate solution to Chandrasekhar's basic problem in radiative transfer. J Quant Spectrosc Radiat Transf 2000;64:109-130.

[68] Siewert CE. A discrete-ordinates solution for radiative-transfer models that include polarizMation effects. J Quant Spectrosc Radiat Transf 2000;64:227254.

[69] Sinnhuber B-M, Sheode N, Sinnhuber M, Chipperfield MP, Feng W. The contribution of anthropogenic bromine emissions to past stratospheric ozone trends: a modelling study. Atmos Chem Phys 2009;9:2863-2871.

[70] Smith RC, Baker KS. Optical properties of the clearest natural waters. Appl Opt 1981;20(2):177-184.

[71] Spitzer D, Dirks RWJ. Contamination of the reflectance of natural waters by solar-induced fluorescence of dissolved organic matter. Appl Opt 1985;24 (4):444-445.

[72] Stamnes K, Tsay SC, Wiscombe W, Jayaweera K. Numerically stable algorithm for discrete-ordinate-method radiative transfer in multiple scattering and emitting layered media. Appl Opt 1988;27:2502-2509.

[73] Stavn RH. Effects of Raman scattering across the visible spectrum in clear ocean water: a Monte Carlo study. Appl Opt 1993;32(33):6853-6863.

[74] Stavn RH, Weidemann AD. Optical modeling of clear ocean light fields: Raman scattering effects. Appl Opt 1988;27(19):4002-4011.

[75] Stavn RH, Weidemann AD. Raman scattering in ocean optics: quantitative assessment of internal radiant emission. Appl Opt 1992;31:1294-1303.
[76] Stedmon CA, Rasmus B. Characterizing dissolved organic matter fluorescence with parallel factor analysis: a tutorial. Limnol Oceanogr: Methods 2008;6:572-579.

[77] Sugihara S, Kishino M, Okami N. Contribution of Raman scattering to upward irradiance in the sea. J Oceanogr Soc Jpn 1984;40:397-404.

[78] Thomas GE, Stamnes K. Radiative transfer in the atmosphere and ocean. Cambridge University Press; 1999.

[79] Traganza ED. Fluorescence excitation and emission spectra of dissolved organic matter in sea water. Bull Mar Sci 1969;19(4):897-904.

[80] van Deelen R. Rotational Raman scattering in the Earth's atmosphere modeling and application to satellite measurements of backscattered sunlight [Ph.D. thesis]. SRON - Netherlands Institute for Space Research; 2007. ISBN 978-90-86591-56-5.

[81] van Deelen R, Landgraf J, Aben I. Multiple elastic and inelastic light scattering in the Earth's atmosphere: a doubling-adding method to include rotational Raman scattering by air. J Quant Spectrosc Radiat Transf 2005;95:309-330.

[82] von Bismarck J. Vibrational Raman scattering of liquid water: quantitative incorporation into a numeric radiative transfer model of the atmosphereocean system and analysis of its impact on remote sensing applications [Ph.D. thesis]. Free University of Berlin; 2015.

[83] Vountas M, Dinter T, Bracher A, Burrows JP, Sierk B. Spectral studies of ocean water with space-borne sensor SCIAMACHY using Differential Optical Absorption Spectroscopy (DOAS). Ocean Sci 2007;3(3):429-440.

[84] Vountas M, Richter A, Wittrock F, Burrows JP. Inelastic scattering in ocean water and its impact on trace gas retrievals from satellite data. Atmos Chem Phys 2003;3:1365-1375.

[85] Vountas M, Rozanov VV, Burrows JP. Ring effect: impact of rotational Raman scattering on radiative transfer in Earth's atmosphere. J Quant Spectrosc Radiat Transf 1998;60:943-961.

[86] Wagner T, Beirle S, Deutschmann T. Three-dimensional simulation of the Ring effect in observations of scattered sun light using Monte Carlo radiative transfer models. Atmos Meas Techn 2009;2:113-124.

[87] Wagner T, Beirle S, Deutschmann T, de Vries MP. A sensitivity analysis of Ring effect to aerosol properties and comparison to satellite observations. Atmos Meas Techn 2010;3:1723-1751.

[88] Wagner T, Deutschmann T, Platt U. Determination of aerosol properties from MAXDOAS observations of the Ring effect. Atmos Meas Techn 2009;2:495-512.

[89] Walrafen GE. Raman spectral studies of the effects of temperature on water structure. J Chem Phys 1967;47(1):114-126.

[90] Wolanin A, Rozanov V, Dinter T, Bracher A. Detecting CDOM fluorescence using high spectrally resolved satellite data: a model study. In: Lohmann G, Meggers H, Unnithan V, Wolf-Gladrow D, Notholt J, Bracher A, editors. Towards an interdisciplinary approach in Earth system science. Cham: Springer International Publishing; 2015. p. 109-121.

[91] Wolanin A, Rozanov VV, Dinter T, Noel S, Vountas M, Burrows JP, et al. Global retrieval of marine chlorophyll fluorescence from high spectrally resolved satellite measurements and its terrestrial application. Remote Sens Environ 2015;166:243-261. 